\title{
Decoherence of quantum Markov semigroups
}

\author{
Rolando Rebolledo ${ }^{1}$ \\ Pontificia Universidad Católica de Chile, Facultad de Matemáticas, Casilla 306, Santiago 22, Chile \\ Received 3 February 2004; received in revised form 2 December 2004; accepted 9 December 2004 \\ Available online 1 April 2005 \\ To the memory of Paul-André Meyer
}

\begin{abstract}
Quantum Decoherence consists in the appearance of classical dynamics in the evolution of a quantum system. This paper focuses on the probabilistic interpretation of this phenomenon, connected with the analysis of classical reductions of a quantum Markov semigroup.

(C) 2005 Elsevier SAS. All rights reserved.
\end{abstract}

\section{Résumé}

La décohérence quantique correspond à l'apparition d'une dynamique classique dans l'évolution d'un système quantique. Cet article fait une interprétation probabiliste de ce phénomène tout en étudiant les réductions classiques des semigroupes Markoviens quantiques.

() 2005 Elsevier SAS. All rights reserved.

\section{Introduction}

For a number of physicists decoherence consists of the dynamical loss of coherences due to the coupled dynamics of an open system and its environment. For a mathematician though, this statement contains numerous unclear concepts claiming for definition. When a quantum system is closed, a single complex separable Hilbert space $\mathfrak{h}$ is used in its description together with a self-adjoint operator $H$ - the Hamiltonian - which is the generator of a unitary group $\left(U_{t}\right)_{t \in \mathbb{R}}$ of operators acting on $\mathfrak{h}$. Thus, $U_{t}=\exp (-\mathrm{i} t H)$ if we assume the Planck constant $\hbar=1$ for simplicity, and given any element $x$ in the von Neumann algebra $\mathfrak{M}=\mathfrak{L}(\mathfrak{h})$ of all bounded linear operators on $\mathfrak{h}$, its evolution is given by a group of automorphisms $\alpha_{t}(x)=U_{t}^{*} x U_{t}, t \in \mathbb{R}$ (Heisenberg picture). The group $\alpha$ naturally determines a group of transformations $\alpha_{*}$ on the predual space $\mathfrak{M}_{*}=\mathfrak{I}_{1}(\mathfrak{h})$ of trace-class operators by the equation $\operatorname{tr}\left(\sigma \alpha_{t}(x)\right)=\operatorname{tr}\left(\alpha_{* t}(\sigma) x\right)$, that is, $\alpha_{* t}(\sigma)=U_{t} \sigma U_{t}^{*},\left(\sigma \in \mathfrak{M}_{*}, x \in \mathfrak{M}\right)$. In particular, the Schrödinger

E-mail address: rrebolle@puc.cl (R. Rebolledo).

1 Research partially supported by FONDECYT grant 1030552 and CONICYT/ECOS exchange program. 
picture which describes the evolution of states, identified here with density matrices $\rho \in \mathfrak{M}_{*}$ which are positive elements with $\operatorname{tr}(\rho)=1$, is $\rho_{t}=\alpha_{* t}(\rho)$.

For an open system, instead, two Hilbert spaces are needed. The previous $\mathfrak{h}$, which we term the system space and the environment space $\mathfrak{h}_{E}$, so that the total dynamics is represented on the space $\mathfrak{H}_{T}=\mathfrak{h} \otimes \mathfrak{h}_{E}$ by a unitary group of generator $H_{T}=H+H_{E}+H_{I}$, where $H_{E}$ (respectively $H_{I}$ ) denotes the Hamiltonian of the environment (respectively, the interaction Hamiltonian). If we want to analyze the reduced dynamics on the space $\mathfrak{h}$, we need to know in addition the state of the environment, that is, a density matrix $\rho_{E} \in \mathfrak{I}_{1}\left(\mathfrak{h}_{E}\right)$ has to be given at the outset. With this density matrix in hands we obtain the Schrödinger picture on $\mathfrak{h}$ by taking a partial trace on the environment variables (denoted $\left.\operatorname{tr}_{\mathrm{E}}\right)$ :

$$
\mathcal{T}_{* t}(\rho)=\operatorname{tr}_{\mathrm{E}}\left(\mathrm{e}^{-\mathrm{i} t H_{T}} \rho \otimes \rho_{E} \mathrm{e}^{\mathrm{i} t H_{T}}\right) .
$$

Or, accordingly, we use $\rho_{E}$ to determine the conditional expectation $\mathbb{E}^{\mathfrak{M}}(\cdot)$ onto $\mathfrak{M}$ to obtain

$$
\mathcal{T}_{t}(x)=\mathbb{E}^{\mathfrak{M}}\left(\mathrm{e}^{\mathrm{i} t H_{T}} x \otimes \mathbf{1}_{E} \mathrm{e}^{-\mathrm{i} t H_{T}}\right),
$$

for $x \in \mathfrak{M}$.

What we now have in hands is a semigroup structure instead of a group. Suppose that an orthonormal basis $\left(e_{n}\right)_{n \in \mathbb{N}}$ is given in $\mathfrak{h}$ so that a density matrix $\rho$ is characterized by its components $\rho(m, n)=\left\langle e_{m}, \rho e_{n}\right\rangle$. Accordingly, $\rho_{t}(m, n)$ denotes the components of $\rho_{t}=\mathcal{T}_{* t}(\rho),(n, m \in \mathbb{N})$. The off-diagonal terms $\rho_{t}(m, n), n \neq m$, are called the coherences. In a rough version, decoherence consists of the disapearance of these terms as time increases, that is $\rho_{t}(m, n) \rightarrow 0$ as $t \rightarrow \infty$. So that, for a large time, the evolution of states becomes essentially described by diagonal matrices which are commutative objects ruled by a classical dynamics. To paraphrase Giulini et al. (see [20]), decoherence is related to the appearance of a classical world in Quantum Theory.

The subject, from the physical point of view, is certainly much more complex than the rough picture drawn before. Several authors pointed out that a most careful analysis of involved time scales should be considered (see [33]). Indeed in numerous physical models, the generator $\mathcal{L}$ of the semigroup $\mathcal{T}$ is obtained by different limiting procedures leading to the so-called master equations (a quantum version of Chapman-Kolmogorov equations), via adequate renormalizations on time and space. The recent book [1] contains a systematic study of these techniques synthesized in the concept of stochastic limit. Thus, for some physicists, decoherence is a phenomenon which precedes the derivation of the so-called Markov approximation of the dynamics. Others, have focused their research on algebraic properties leading to a definition and main properties of decoherence in the Heisenberg picture (see [4]), while another group looks for the physical causes of decoherence, mainly attributed to interactions of the system with the boundaries of the cavity in which it is contained (see [13]). The debate has been reinforced by experimental results allowing to observe the decay of coherences for a given initial coherent superposition of two pure states (see the reports of the Haroche's group at the ENS in Paris [7], and that of the Wineland's group in Boulder [28]).

The physical problem of decoherence is undoubtedly passionating and its discussion leads directly to philosophical arguments about the foundations of Quantum Mechanics. We do not pretend here to enter that arena. We address a much more modest problem which can be easily stated within a well known mathematical framework, we refer to Quantum Markov Theory.

We separate two aspects in the analysis of decoherence. Firstly, the question of the existence of an Abelian subalgebra, generated by a given self-adjoint operator, which remains invariant under the action of a given quantum Markov semigroup (QMS). Thus, the restriction of the semigroup to that algebra will provide a classical Markov semigroup. Secondly, we focus on the asymptotic behavior of a QMS. Our goal in that part is to analyze structure properties of the semigroup leading to a classical limit behavior. Preferred by physicists to construct mathematical models of open quantum systems, the generator will be our main tool to analyze decoherence of a quantum Markov semigroup. 


\section{Quantum Markov Semigroups}

A Quantum Markov Semigroup (QMS) arises as the natural non-commutative extension of the well known concept of Markov semigroup defined on a classical probability space and represents the loss-memory evolution of a microscopic system in accordance with the quantum uncertainty principle. The roots of the theory go back to the first researches on the so called open quantum systems (for an account see [2]), and have found its main non-commutative tools in much older abstract results like the characterization of complete positive maps due to Stinespring (see [30,31]). Indeed, complete positivity contains a deep probabilistic notion expressed in the language of operator algebras. In many respects it is the core of mathematical properties of (regular versions of) conditional expectations. Thus, complete positivity appears as a keystone in the definition of a QMS. Moreover, in classical Markov Theory, topology plays a fundamental role which goes from the basic setting of the space of states up to continuity properties of the semigroup. In particular, Feller property allows to obtain stronger results on the qualitative behavior of a Markov semigroup. In the non-commutative framework, Feller property is expressed as a topological and algebraic condition. Namely, a classical semigroup satisfying the Feller property on a locally compact state space leaves invariant the algebra of continuous functions with compact support, which is a particular example of a $C^{*}$-algebra. The basic ingredients to start with a non-commutative version of Markov semigroups are then two: firstly, a ${ }^{*}$-algebra $\mathfrak{A}$, that means an algebra endowed with an involution ${ }^{*}$ which satisfies $\left(a^{*}\right)^{*}=a$, $(a b)^{*}=b^{*} a^{*}$, for all $a, b \in \mathfrak{A}$, in addition we assume that the algebra contains a unit $\mathbf{1}$; and secondly, we need a semigroup of completely positive maps from $\mathfrak{A}$ to $\mathfrak{A}$ which preserves the unit. We will give a precise meaning to this below. We remind that positive elements of the *-algebra are of the form $a^{*} a,(a \in \mathfrak{A})$. A state $\varphi$ is a linear $\operatorname{map} \varphi: \mathfrak{A} \rightarrow \mathbb{C}$ such that $\varphi(\mathbf{1})=1$, and $\varphi\left(a^{*} a\right) \geqslant 0$ for all $a \in \mathfrak{A}$.

Definition 1. Let $\mathfrak{A}$ be a ${ }^{*}$-algebra and $\mathcal{P}: \mathfrak{A} \rightarrow \mathfrak{A}$ a linear map. $\mathcal{P}$ is completely positive if for any finite collection $a_{1}, \ldots, a_{n}, b_{1}, \ldots, b_{n}$ of elements of $\mathfrak{A}$ the element

$$
\sum_{i, j} a_{i}^{*} \mathcal{P}\left(b_{i}^{*} b_{j}\right) a_{j}
$$

is positive.

Throughout this paper, we will restrict our *-algebras to the significant cases of $C^{*}$ algebras and von Neumann algebras of operators on a complex separable Hilbert space $\mathfrak{h}$. The symbol $\mathfrak{M}$ will be used to denote a generic von Neumann algebra, while $\mathfrak{B}$ will be assigned to a $C^{*}$-algebra. Moreover, we always assume that our $C^{*}$-algebra $\mathfrak{B}$ contains a unit 1 . In this case states are elements of the dual $\mathfrak{B}^{*}$ of $\mathfrak{B}$. A state $\varphi$ is pure if the only positive linear functionals majorized by $\varphi$ are of the form $\lambda \varphi$ with $0 \leqslant \lambda \leqslant 1$. For an Abelian $C^{*}$-algebra, the set of pure states coincides with that of all characters, also called spectrum of the algebra (see [5], Proposition 2.3.27, p. 62). A character $\varphi$ of an Abelian $C^{*}$-algebra $\mathfrak{A}$ is a state which satisfies $\varphi(a b)=\varphi(a) \varphi(b)$, for all $a, b \in \mathfrak{A}$; the set of all these elements is usually denoted $\sigma(\mathfrak{A})$ (for spectrum) or $P_{\mathfrak{A}}$ (for pure states).

If $\mathfrak{M}$ is a von Neumann algebra, its predual is denoted $\mathfrak{M}_{*}$. The predual contains in particular all the normal states. As a rule, we will only deal with normal states $\varphi$ for which there exists a density matrix $\rho$, that is, a positive trace-class operator of $\mathfrak{h}$ with unit trace, such that $\varphi(a)=\operatorname{tr}(\rho a)$ for all $a \in \mathcal{A}$.

Definition 2. A quantum sub-Markov semigroup, or quantum dynamical semigroup (QDS) on a *-algebra $\mathfrak{A}$ which has a unit $\mathbf{1}$, is a one-parameter family $\mathcal{T}=\left(\mathcal{T}_{t}\right)_{t \in \mathbb{R}_{+}}$of linear maps of $\mathfrak{A}$ into itself satisfying

(M1) $\mathcal{T}_{0}(x)=x$, for all $x \in \mathfrak{A}$;

(M2) Each $\mathcal{T}_{t}(\cdot)$ is completely positive;

(M3) $\mathcal{T}_{t}\left(\mathcal{T}_{s}(x)\right)=\mathcal{T}_{t+s}(x)$, for all $t, s \geqslant 0, x \in \mathfrak{A}$;

(M4) $\mathcal{T}_{t}(\mathbf{1}) \leqslant \mathbf{1}$ for all $t \geqslant 0$. 
A quantum dynamical semigroup is called quantum Markov (QMS) if $\mathcal{T}_{t}(\mathbf{1})=\mathbf{1}$ for all $t \geqslant 0$.

If $\mathfrak{A}$ is a $C^{*}$-algebra, then a quantum dynamical semigroup is uniformly (or norm) continuous if it additionally satisfies

(M5) $\lim _{t \rightarrow 0} \sup _{\|x\| \leqslant 1}\left\|\mathcal{T}_{t}(x)-x\right\|=0$.

This is a very strong continuity condition which is sometimes replaced by the so called Feller continuity condition (M5F) $\lim _{t \rightarrow 0}\left\|\mathcal{T}_{t}(x)-x\right\|=0$, for all $x \in \mathfrak{A}$.

A quantum Markov semigroup satisfying (M5F) will be called quantum Feller.

If $\mathfrak{A}$ is a von Neumann algebra, (M5) is usually replaced by the weaker condition

(M5 $\sigma$ ) For each $x \in \mathfrak{A}$, the map $t \mapsto \mathcal{T}_{t}(x)$ is $\sigma$-weak continuous on $\mathfrak{A}$, and $\mathcal{T}_{t}(\cdot)$ is normal or $\sigma$-weak continuous.

The generator $\mathcal{L}$ of the semigroup $\mathcal{T}$ is then defined in the $w^{*}$ or $\sigma$-weak sense. That is, its domain $D(\mathcal{L})$ consists of elements $x$ of the algebra for which the $w^{*}$-limit of $t^{-1}\left(\mathcal{T}_{t}(x)-x\right)$ exists as $t \rightarrow 0$. This limit is denoted then $\mathcal{L}(x)$.

The predual semigroup $\mathcal{T}_{*}$ is defined on $\mathfrak{M}_{*}$ as $\mathcal{T}_{* t}(\varphi)(x)=\varphi\left(\mathcal{T}_{t}(x)\right)$ for all $t \geqslant 0, x \in \mathfrak{M}, \varphi \in \mathfrak{M}_{*}$. Its generator is denoted $\mathcal{L}_{*}$.

It is worth noticing that the generator $\mathcal{L}$ is often known indirectly through sesquilinear forms and the so called Master Equations in the case $\mathfrak{M}=\mathfrak{L}(\mathfrak{h})$. These equations are expressed in terms of density matrices $\rho \in \mathfrak{I}_{1}(\mathfrak{h})$, the space of trace-class operators in $\mathfrak{L}(\mathfrak{h})$, and they correspond to a non-commutative version of ChapmanKolmogorov's equations:

$$
\left\{\begin{array}{l}
\frac{\mathrm{d}}{\mathrm{d} t}\left\langle v, \rho_{t} u\right\rangle=\mathfrak{L}_{*}\left(\rho_{t}\right)(v, u), \\
\rho_{0}=\rho,
\end{array}\right.
$$

$u, v \in \mathfrak{h}, \rho_{t}$ corresponds to the action of the predual semigroup on $\rho$ at time $t$ and $(u, v) \mapsto \mathfrak{L}_{*}(\rho)(v, u)$ corresponds to a sesquilinear form which is linear in $u \in \mathfrak{h}$, and antilinear in $v \in \mathfrak{h}$.

\subsection{The generator in the $C^{*}$-case}

A quantum Markov semigroup is norm-continuous if and only if its generator $\mathcal{L}(\cdot)$ is a bounded operator on $\mathfrak{B}$.

In [10], Christensen and Evans provided an expression for the infinitesimal generator $\mathcal{L}$ of a norm-continuous quantum dynamical semigroup defined on a $C^{*}$-algebra, extending previous results obtained by Lindblad [25], Gorini, Kossakowski and Sudarshan [21]. We recall their result here below.

Suppose that $\mathcal{T}$ is a norm-continuous quantum dynamical semigroup on $\mathfrak{B}$ and denote $\overline{\mathfrak{B}}$ the $\sigma$-weak closure of the $C^{*}$-algebra $\mathfrak{B}$. Then there exists a completely positive map $\Psi: \mathfrak{B} \rightarrow \overline{\mathfrak{B}}$ and an operator $G \in \overline{\mathfrak{B}}$ such that the generator $\mathcal{L}(\cdot)$ of the semigroup is given by

$$
\mathcal{L}(x)=G^{*} x+\Psi(x)+x G \quad(x \in \mathfrak{B}) .
$$

The map $\Psi$ can be represented by means of Stinespring Theorem [31] as follows. There exists a representation $(\mathfrak{k}, \pi)$ of the algebra $\mathfrak{B}$ and a bounded operator $V$ from $\mathfrak{h}$ to the Hilbert space $\mathfrak{k}$ such that

$$
\Psi(x)=V^{*} \pi(x) V \quad(x \in \mathfrak{B}) .
$$


Notice that $\Psi(\mathbf{1})=V^{*} V=-\left(G^{*}+G\right)=-2 \Re(G) \in \overline{\mathfrak{B}}$, where $\Re(G)$ denotes the real part of $G$, since $\mathcal{L}(\mathbf{1})=$ 0 . So that, if we call $H$ the selfadjoint operator $2^{-1} \mathrm{i}\left(G-G^{*}\right)=-\Im(G) \in \overline{\mathfrak{B}}$, where $\Im(G)$ stands for the imaginary part of $G$, then $\mathcal{L}(\cdot)$ can also be written as

$$
\mathcal{L}(x)=\mathrm{i}[H, x]-\frac{1}{2}\left(V^{*} V x-2 V^{*} \pi(x) V-x V^{*} V\right) \quad(x \in \mathfrak{B}) .
$$

The representation of $\mathcal{L}(x)$ in terms of $G$ and $\Psi$ is certainly not unique.

\subsection{The generator in the von Neumann case}

Consider a von Neumann algebra $\mathfrak{M}$ on the Hilbert space $\mathfrak{h}$. The representation of the generator $\mathcal{L}(\cdot)$ of a norm continuous QMS on $\mathfrak{M}$ is then improved as follows. There exists a set of operators $\left(L_{k}\right)_{k \in \mathbb{N}}$ such that $L=\sum_{k} L_{k}^{*} L_{k}$ is a bounded operator in $\mathfrak{M}$ and $\sum_{k} L_{k}^{*} x L_{k} \in \mathfrak{M}$ whenever $x \in \mathfrak{M}$ and a selfadjoint operator $H=H^{*} \in \mathfrak{M}$ such that

$$
\mathcal{L}(x)=\mathrm{i}[H, x]-\frac{1}{2} \sum_{k}\left(L_{k}{ }^{*} L_{k} x-2 L_{k}{ }^{*} x L_{k}+x L_{k}{ }^{*} L_{k}\right) .
$$

We recover the expression (2) if we put

$$
G=-\mathrm{i} H-\frac{1}{2} \sum_{k} L_{k}^{*} L_{k} ; \quad \Psi(x)=\sum_{k} L_{k}^{*} x L_{k} .
$$

\subsection{The case of a form-generator}

In most of applications $\mathcal{L}(\cdot)$ is not known as an operator directly but through a sesquilinear form. To discuss this case we restrict ourselves to the von Neumann algebra $\mathfrak{M}=\mathfrak{L}(\mathfrak{h})$ and we rephrase, for easier reference the crucial result which allows to construct a quantum dynamical semigroup starting from a generator given as a sesquilinear form. For further details on this matter we refer to [14], Section 3.3, see also [9].

Let $G$ and $L_{\ell}(\ell \geqslant 1)$ be operators in $\mathfrak{h}$ which satisfy the following hypothesis:

(H-min) $G$ is the infinitesimal generator of a strongly continuous contraction semigroup in $\mathfrak{h}, D(G)$ is contained in $D\left(L_{\ell}\right)$, for all $\ell \geqslant 1$, and, for all $u, v \in D(G)$, we have

$$
\langle G v, u\rangle+\sum_{\ell=1}^{\infty}\left\langle L_{\ell} v, L_{\ell} u\right\rangle+\langle v, G u\rangle=0
$$

Under the above assumption (H-min), for each $x \in \mathfrak{L}(\mathfrak{h})$ let $\mathfrak{L}(x)$ be the sesquilinear form with domain $D(G) \times D(G)$ defined by

$$
\mathfrak{L}(x)(v, u)=\langle G v, x u\rangle+\sum_{\ell=1}^{\infty}\left\langle L_{\ell} v, x L_{\ell} u\right\rangle+\langle v, x G u\rangle .
$$

It is well-known (see e.g. [11], Section 3, [14], Section 3.3) that, given a domain $D \subseteq D(G)$, which is a core for $G$, it is possible to built up a quantum dynamical semigroup, called the minimal QDS, satisfying the equation:

$$
\left\langle v, \mathcal{T}_{t}(x) u\right\rangle=\langle v, x u\rangle+\int_{0}^{t} \mathfrak{L}\left(\mathcal{T}_{s}(x)\right)(v, u) \mathrm{d} s,
$$

for $u, v \in D$. 
This equation, however, in spite of the hypothesis (H-min) and the fact that $D$ is a core for $G$, does not necessarily determine a unique semigroup. The minimal QDS is characterized by the following property: for any $w^{*}$-continuous family $\left(\mathcal{T}_{t}\right)_{t} \geqslant 0$ of positive maps on $\mathfrak{L}(\mathfrak{h})$ satisfying $(8)$ we have $\mathcal{T}_{t}^{(\min )}(x) \leqslant \mathcal{T}_{t}(x)$ for all positive $x \in \mathfrak{L}(\mathfrak{h})$ and all $t \geqslant 0$ (see e.g. [11]; [14], Theorem 3.22; [22], Theorem 3.3.3, p. 86). If the minimal QDS is Markov, that is $\mathcal{T}_{t}^{(\mathrm{min})}(\mathbf{1})=\mathbf{1}$, for all $t \geqslant 0$, then it is the unique solution to (8). One may test that condition on the formal generator through the equation $\mathfrak{L}(\mathbf{1})(v, u)=0$ for all $u, v \in D$. Throughout this paper we will implicitly assume that formal generators satisfy the Markov property, and we will then refer to the unique minimal solution of (8) as the minimal quantum Markov semigroup. For a discussion on sufficient conditions for this property to hold the reader is addressed to $[8,22]$.

The assumed density of $D$ implies that the minimal quantum Markov semigroup - which we simply denote $\mathcal{T}$ from now on - possess a densely defined generator $\mathcal{L}$, its domain $D(\mathcal{L}$ being given by all elements $x \in \mathfrak{L}(\mathfrak{h})$ for which the map $(u, v) \mapsto \mathfrak{L}(x)(u, v)$ is norm-continuous on the product Hilbert space $\mathfrak{h} \times \mathfrak{h}$. And, for any $x \in D(\mathcal{L}), \mathcal{L}(x)$ is given by $\langle v, \mathcal{L}(x) u\rangle=\mathfrak{L}(x)(u, v), u, v \in \mathfrak{h}$, after extending $\mathfrak{L}(x)$ from the dense set $D \times D$ to all of $\mathfrak{h} \times \mathfrak{h}$ by continuity. Also, Proposition 3.1.6 in [5], shows that $\mathcal{L}$ is $w^{*}$-closed. Moreover, the predual semigroup $\mathcal{T}_{*}$ is now defined on the predual space of $\mathfrak{L}(\mathfrak{h})$ which is $\mathfrak{I}_{1}(\mathfrak{h})$, the Banach space of trace-class operators: $\operatorname{tr}\left(\mathcal{T}_{* t}(\rho) x\right)=\operatorname{tr}\left(\rho \mathcal{T}_{t}(x)\right),\left(\rho \in \mathfrak{I}_{1}(\mathfrak{h}), x \in \mathfrak{L}(\mathfrak{h})\right)$. So that, $\mathcal{L}_{*}\left(D\left(\mathcal{L}_{*}\right)\right) \subset \mathfrak{I}_{1}(\mathfrak{h})$ and, as Davies proved in [11] (see also [15]), the dense set $\{|u\rangle\langle v| ; u, v \in D\}$ is included in the domain $D\left(\mathcal{L}_{*}\right)$ of the predual semigroup generator. This predual semigroup $\mathcal{T}_{*}$ is weakly-continuous. This property, together with complete positivity, imply that it is also a strongly continuous semigroup of bounded linear operators (a so called $C_{0}$-semigroup) since $\mathfrak{I}_{1}(\mathfrak{h}$ ) is a Banach space and Corollary 2.5 in [29] holds. As a result, $\mathcal{L}_{*}$ is a closed operator too.

\subsection{Examples of generators}

\subsubsection{The quantum damped harmonic oscillator}

In this case the Hilbert space used to represent the system is $\mathfrak{h}=\ell^{2}(\mathbb{N})$ with its canonical orthonormal basis $\left(e_{n}\right)_{n \in \mathbb{N}} ; \mathfrak{M}=\mathfrak{L}(\mathfrak{h})$. We use the customary notations for annihilation $(a)$, creation $\left(a^{\dagger}\right)$ and number $(N)$ operators. The physical model corresponds to an atom which traverses an ideal resonator (a high quality cavity), its energy can be in two levels only (a so called two-level atom). Excitations of a mode of the quantized radiation field in the resonator correspond to photons which stay in the cavity, they have a finite life-time and they interact with the incident atom. The physical description of the dynamics have been obtained by different approximation procedures (weak coupling limit, coarse graining) which end in a Master Equation containing the (formal) generator of a quantum Markov semigroup. Here we start from that formal generator, the reader interested in its physical derivation is addressed to any textbook on Quantum Optics, here we use the presentation of B.E. Englert and G. Morigi in page 55 of the collective book [33]. Let introduce the physical parameters: A denotes the energy decay rate in the cavity; $v$, the number of thermal excitations; $\omega$, the natural (circular) frequency. The form-generator of the semigroup is given by the (formal) expression

$$
\mathcal{L}(x)=\mathrm{i}[\omega N, x]-\frac{1}{2} A(v+1)\left(a^{\dagger} a x-2 a^{\dagger} x a+x a^{\dagger} a\right)-\frac{1}{2} A v\left(a a^{\dagger} x-2 a x a^{\dagger}+x a a^{\dagger}\right),
$$

for $x$ in a dense subset of $\mathfrak{M}$, which is the common domain of $a$ and $a^{\dagger}$.

\subsubsection{The quantum Brownian motion}

Let $\mathfrak{h}=L^{2}\left(\mathbb{R}^{d} ; \mathbb{C}\right)$ and $\mathfrak{M}=\mathfrak{L}(\mathfrak{h})$. We consider here another version of the harmonic oscillator like in [27], Chapter III (see also [3]). Though this is an extension of the previous example, the dimension $d$ plays here an important role in the analysis of ergodic properties of the semigroup. A Quantum Brownian Motion means for us a 
quantum Markov process with associated semigroup $\mathcal{T}$ on $\mathfrak{M}$ which is the minimal semigroup (see $[8,14]$ and the references therein) with form-generator

$$
\mathfrak{L}(x)=-\frac{1}{2} \sum_{j=1}^{d}\left(a_{j} a_{j}^{\dagger} x-2 a_{j} x a_{j}^{\dagger}+x a_{j} a_{j}^{\dagger}\right)-\frac{1}{2} \sum_{j=1}^{d}\left(a_{j}^{\dagger} a_{j} x-2 a_{j}^{\dagger} x a_{j}+x a_{j}^{\dagger} a_{j}\right),
$$

where $a_{j}^{\dagger}, a_{j}$ are the creation and annihilation operators

$$
a_{j}=\left(q_{j}+\partial_{j}\right) / \sqrt{2}, \quad a_{j}^{\dagger}=\left(q_{j}-\partial_{j}\right) / \sqrt{2},
$$

$\partial_{j}$ being the partial derivative with respect to the $j$ th coordinate $q_{j}$.

\subsubsection{The quantum exclusion semigroup}

The generator of this example is constructed via a second quantization procedure. Consider first a self-adjoint bounded operator $H_{0}$ defined on a separable complex Hilbert space $\mathfrak{h}_{0} . H_{0}$ will be thought of as describing the dynamics of a single fermionic particle. We assume that there is an orthonormal basis $\left(\psi_{n}\right)_{n \in \mathbb{N}}$ of eigenvectors of $H_{0}$, and denote $E_{n}$ the eigenvalue of $\psi_{n}(n \in \mathbb{N})$. The set of all finite subsets of $\mathbb{N}$ is denoted $\mathfrak{P}_{f}(\mathbb{N})$ and for any $\Lambda \in \mathfrak{P}_{f}(\mathbb{N})$, we denote $\mathfrak{h}_{0}^{\Lambda}$ the finite-dimensional Hilbert subspace of $\mathfrak{h}_{0}$ generated by the vectors $\left(\psi_{n} ; n \in \Lambda\right)$. To deal with a system of infinite particles we introduce the fermionic Fock space $\mathfrak{h}=\Gamma_{f}\left(\mathfrak{h}_{0}\right)$ associated to $\mathfrak{h}_{0}$ whose construction we recall briefly (see [6] for full detail).

The Fock space associated to $\mathfrak{h}_{0}$ is the direct sum

$$
\Gamma\left(\mathfrak{h}_{0}\right)=\bigoplus_{n \in \mathbb{N}} \mathfrak{h}_{0}^{\otimes n},
$$

where $\mathfrak{h}_{0}^{\otimes n}$ is the $n$-fold tensor product of $\mathfrak{h}_{0}$, with the convention $\mathfrak{h}_{0}^{\otimes 0}=\mathbb{C}$. Define an operator $\mathbf{P}_{\mathrm{a}}$ on the Fock space as follows,

$$
\mathbf{P}_{\mathrm{a}}\left(f_{1} \otimes f_{2} \otimes \cdots \otimes f_{n}\right)=\frac{1}{n !} \sum_{\pi} \varepsilon_{\pi} f_{\pi_{1}} \otimes \cdots \otimes f_{\pi_{n}} .
$$

The sum is over all permutations $\pi:\{1, \ldots, n\} \rightarrow\left\{\pi_{1}, \ldots, \pi_{n}\right\}$ of the indices and $\varepsilon_{\pi}$ is 1 if $\pi$ is even and -1 if $\pi$ is odd. Define the anti-symmetric tensor product on the Fock space as $f_{1} \wedge \cdots \wedge f_{n}=\mathbf{P}_{\mathrm{a}}\left(f_{1} \otimes f_{2} \otimes \cdots \otimes f_{n}\right)$. In this manner, the Fermi-Fock space $\mathfrak{h}$ is obtained as

$$
\mathfrak{h}=\Gamma_{f}\left(\mathfrak{h}_{0}\right)=\mathbf{P}_{\mathrm{a}}\left(\bigoplus_{n \in \mathbb{N}} \mathfrak{h}_{0}^{\otimes n}\right)=\bigoplus_{n \in \mathbb{N}} \mathfrak{h}_{0}^{\wedge n}
$$

We follow [6] to introduce the so-called fermionic Creation $b^{\dagger}(f)$ and Annihilation $b(f)$ operators on $\mathfrak{h}$, associated to a given element $f$ of $\mathfrak{h}_{0}$. Firstly, on $\Gamma\left(\mathfrak{h}_{0}\right)$ we define $a(f)$ and $a^{\dagger}(f)$ by initially setting $a(f) \psi^{(0)}=0$, $a^{\dagger}(f) \psi^{(0)}=f$, for $\psi=\left(\psi^{(0)}, \psi^{(1)}, \ldots\right) \in \Gamma\left(\mathfrak{h}_{0}\right)$ with $\psi^{(j)}=0$ for all $j \geqslant 1$, and

$$
\begin{aligned}
& a^{\dagger}(f)\left(f_{1} \otimes \cdots \otimes f_{n}\right)=\sqrt{n+1} f \otimes f_{1} \otimes \cdots \otimes f_{n}, \\
& a(f)\left(f_{1} \otimes \cdots \otimes f_{n}\right)=\sqrt{n}\left\langle f, f_{1}\right\rangle f_{2} \otimes f_{3} \otimes \cdots \otimes f_{n} .
\end{aligned}
$$

Finally, define annihilation and creation on $\Gamma_{f}\left(\mathfrak{h}_{0}\right)$ as $b(f)=\mathbf{P}_{\mathrm{a}} a(f) \mathbf{P}_{\mathrm{a}}$ and $b^{\dagger}(f)=\mathbf{P}_{\mathrm{a}} a^{\dagger}(f) \mathbf{P}_{\mathrm{a}}$. These operators satisfy the Canonical Anti-commutation Relations (CAR) on the Fermi-Fock space:

$$
\begin{aligned}
& \{b(f), b(g)\}=0=\left\{b^{\dagger}(f), b^{\dagger}(g)\right\}, \\
& \left\{b(f), b^{\dagger}(g)\right\}=\langle f, g\rangle \mathbf{1},
\end{aligned}
$$

for all $f, g \in \mathfrak{h}_{0}$, where we use the notation $\{A, B\}=A B+B A$ for two operators $A$ and $B$. 
Moreover, $b(f)$ and $b^{\dagger}(g)$ have bounded extensions to the whole space $\mathfrak{h}$ since $\|b(f)\|=\|f\|=\left\|b^{\dagger}(f)\right\|$.

To simplify notations, we write $b_{n}^{\dagger}=b^{\dagger}\left(\psi_{m}\right)$ (respectively $\left.b_{n}=b\left(\psi_{n}\right)\right)$ the creation (respectively annihilation) operator associated with $\psi_{n}$ in the space $\mathfrak{h}_{0},(n \in \mathbb{N})$.

The $C^{*}$-algebra generated by $\mathbf{1}$ and all the $b(f), f \in \mathfrak{h}_{0}$, is denoted $\mathfrak{A}\left(\mathfrak{h}_{0}\right)$ and it is known as the canonical $C A R$ algebra.

Remark 1. The algebra $\mathfrak{A}\left(\mathfrak{h}_{0}\right)$ is the unique, up to *-isomorphism, $C^{*}$-algebra generated by elements $b(f)$ satisfying the anti-commutation relations over $\mathfrak{h}_{0}$ (see e.g. [6], Theorem 5.2.5).

Remark 2. It is worth mentioning that the family $\left(b(f), b^{\dagger}(g) ; f, g \in \mathfrak{h}_{0}\right)$ is irreducible on $\mathfrak{h}$, that is, the only operators which commute with this family are the scalar multiples of the identity ([6], Proposition 5.2.2). Clearly, the same property is satisfied by the family $\left(b_{n}, b_{n}^{\dagger} ; n \in \mathbb{N}\right)$, since $\left(\psi_{n}\right)_{n \in \mathbb{N}}$ is an orthonormal basis of $\mathfrak{h}_{0}$.

Remark 3. The algebra $\mathfrak{A}\left(\mathfrak{h}_{0}\right)$ is the strong closure of $\mathfrak{D}=\bigcup_{\Lambda \in \mathfrak{P}_{f}(\mathbb{N})} \mathfrak{A}\left(\mathfrak{h}_{0}^{\Lambda}\right)$ (see [6], Proposition 5.2.6), this is the quasi-local property. Moreover, the finite dimensional algebras $\mathfrak{A}\left(\mathfrak{h}_{0}^{\Lambda}\right)$ are isomorphic to algebras of matrices with complex components.

An element $\eta$ of $\{0,1\}^{\mathbb{N}}$ will be called a configuration of particles. For each $n, \eta(n)$ will take the value 1 or 0 depending on whether the $n$-th site has been occupied by a particle in the configuration $\eta$. In other terms, we say that the site $n$ is occupied by the configuration $\eta$ if $\eta(n)=1$. We denote $\mathbf{S}$ the set of configurations $\eta$ with a finite number of 1's, that is $\sum_{n} \eta(n)<\infty$. Each $\eta \in \mathbf{S}$ is then identifiable to the characteristic function $1_{\left\{s_{1}, \ldots, s_{m}\right\}}$ of a finite subset of $\mathbb{N}$, which, in addition, we will suppose ordered as $0 \leqslant s_{1}<s_{2}<\cdots<s_{m}$. For simplicity we write $1_{k}$ the configuration $1_{\{k\}},(k \in \mathbb{N})$. Furthermore, we define

$$
\begin{aligned}
& \mathbf{b}^{\dagger}(\eta)=b_{s_{m}}^{\dagger} b_{s_{m-1}}^{\dagger} \ldots b_{s_{1}}^{\dagger}, \\
& \mathbf{b}(\eta)=b_{s_{m}} b_{s_{m-1}} \ldots b_{s_{1}},
\end{aligned}
$$

for all $\eta=1_{\left\{s_{1}, \ldots, s_{m}\right\}}$. Clearly, $\mathbf{b}^{\dagger}\left(1_{k}\right)=b_{k}^{\dagger}, \mathbf{b}\left(1_{k}\right)=b_{k}(k \in \mathbb{N})$.

To obtain a cyclic representation of $\mathfrak{A}\left(\mathfrak{h}_{0}\right)$ we call $|0\rangle$ the vacuum vector in $\mathfrak{h}$, and $|\eta\rangle=\mathbf{b}^{\dagger}(\eta)|0\rangle(\eta \in \mathbf{S})$. Then $(|\eta\rangle, \eta \in \mathbf{S})$ is an orthonormal basis of $\mathfrak{h}$. In this manner, any $x \in \mathfrak{A}\left(\mathfrak{h}_{0}\right)$ can be represented as an operator in $\mathfrak{L}(\mathfrak{h})$. Moreover, call $\mathfrak{v}$ the vector space spanned by $(|\eta\rangle, \eta \in \mathbf{S})$.

An elementary computation based on the C.A.R. shows that for any $\eta, \zeta \in \mathbf{S}$, it holds

$$
\begin{aligned}
& b_{k}^{\dagger}|\eta\rangle=(1-\eta(k))\left|\eta+1_{k}\right\rangle, \\
& b_{k}|\eta\rangle=\eta(k)\left|\eta-1_{k}\right\rangle \quad(k \in \mathbb{N}) .
\end{aligned}
$$

We assume in addition that $H_{0}$ is bounded from below, so that there exists $b \in \mathbb{R}$ such that $b<E_{n}$ for all $n \in \mathbb{N}$. Then, the second quantization of $H_{0}$ becomes a self-adjoint operator $H$ acting on $\mathfrak{h}$, with domain $D(H)$ which includes $\mathfrak{v}$ and can formally be written as

$$
H=\sum_{n} E_{n} b_{n}^{\dagger} b_{n}
$$

It is worth mentioning that the restriction $H^{\Lambda}=\sum_{n \in \Lambda} E_{n} b_{n}^{\dagger} b_{n}$ of $H$ to each space $\Gamma_{f}\left(\mathfrak{h}_{0}^{\Lambda}\right)$ is an element of the algebra $\mathfrak{A}\left(\mathfrak{h}_{0}^{\Lambda}\right), \Lambda \in \mathfrak{P}_{f}(\mathbb{N})$, so that $H^{\Lambda}$ is a bounded operator. Moreover, for each $\| H|\eta\rangle-H^{\Lambda}|\eta\rangle \| \rightarrow 0$ as $\Lambda$ increases to $\mathbb{N}$, for each $\eta \in \mathbf{S}$.

The transport of a particle from a site $i$ to a site $j$, at a rate $\gamma_{i, j}$ is described by an operator $L_{i, j}$ defined as

$$
L_{i, j}=\sqrt{\gamma_{i, j}} b_{j}^{\dagger} b_{i}
$$


This corresponds to the action of a reservoir on the system of fermionic particles pushing them to jump between different sites. Each operator $L_{i, j}$ is an element of $\mathfrak{A}\left(\mathfrak{h}_{0}\right)$ and $\left\|L_{i, j}\right\|=\sqrt{\gamma_{i, j}}$. We additionally assume that

$$
\sup _{i} \sum_{j} \gamma_{i, j}<\infty \text {. }
$$

Now, for each $\Lambda \in \mathfrak{P}_{f}(\mathbb{N})$ and $x \in \mathfrak{A}\left(\mathfrak{h}_{0}\right)$, define

$$
\varphi_{\Lambda}(x)=\sum_{i, j \in \Lambda} L_{i, j}{ }^{*} x L_{i, j}
$$

$\varphi_{\Lambda}: \mathfrak{A}\left(\mathfrak{h}_{0}\right) \rightarrow \mathfrak{A}\left(\mathfrak{h}_{0}\right)$ is a completely positive map. Moreover, for each vector $|\eta\rangle$ of the orthonormal basis in $\mathfrak{h}$,

$$
\begin{aligned}
\| \varphi_{\Lambda}(x)|\eta\rangle \| & \leqslant \sum_{i, j \in \Lambda} \| L_{i, j}{ }^{*} x L_{i, j}|\eta\rangle\left\|=\sum_{i, j \in \Lambda} \eta(i)(1-\eta(j)) \sqrt{\gamma_{i, j}}\right\| L_{i, j}{ }^{*} x\left|\eta-1_{i}+1_{j}\right\rangle \| \\
& \leqslant \sum_{i, j \in \Lambda} \eta(i)(1-\eta(j)) \gamma_{i, j}\|x\| \leqslant\left(\sup _{i} \sum_{j} \gamma_{i, j}\right)\|x\| .
\end{aligned}
$$

So that $\| \varphi_{\Lambda}(x)|\eta\rangle \|$ is uniformly bounded as $\Lambda$ run over $\mathfrak{P}_{f}(\mathbb{N})$. Moreover,

$$
\sum_{i, j} \| L_{i, j}^{*} x L_{i, j}|\eta\rangle \|<\infty
$$

so that $\left\|\left(\varphi_{\Lambda}(x)-\varphi(x)\right) u\right\| \rightarrow 0$ for all $u \in \mathfrak{v}$ as $\Lambda \uparrow \mathbb{N}$, where the operator

$$
\varphi(x)=\sum_{i, j} L_{i, j}{ }^{*} x L_{i, j},
$$

is defined on the dense manifold $\mathfrak{v}$ for all $x \in \mathfrak{A}\left(\mathfrak{h}_{0}\right)$.

As a result, $\varphi_{\Lambda}(\mathbf{1})$ converges in the same sense to $\varphi(\mathbf{1})=\sum_{i, j} L_{i, j}{ }^{*} L_{i, j}$.

To summarize, the generator of the QMS in this case is given in the form (5):

$$
\mathcal{L}(x)=\mathrm{i}[H, x]-\frac{1}{2} \sum_{i, j}\left(L_{i, j}{ }^{*} L_{i, j} x-2 L_{i, j}{ }^{*} x L_{i, j}+x L_{i, j}{ }^{*} L_{i, j}\right),
$$

with $H$ and $L_{i, j}$ introduced in (18), (19), $x \in \mathfrak{A}\left(\mathfrak{h}_{0}\right)$ and $\mathfrak{v}$ is a core domain for $\mathcal{L}(x)$.

\section{The appearance of classical Markov semigroups}

Given a quantum Markov semigroup $\mathcal{T}$ and a self-adjoint operator $K$, this section studies different conditions on the generator of the semigroup to leave invariant the Abelian algebra generated by $K$.

Definition 3. We say that a completely positive map $\mathcal{P}$ defined on $\mathfrak{B}$ is reduced by an Abelian *-subalgebra $\mathfrak{A}$ if $\mathfrak{A} \subseteq \mathfrak{B}$ is invariant under the action of $\mathcal{P}$.

Analogously, a quantum dynamical (resp. Markov) semigroup $\left(\mathcal{T}_{t}\right)_{t \in \mathbb{R}^{+}}$defined on $\mathfrak{B}$ is reduced by $\mathfrak{A}$ if $\mathfrak{A} \subseteq \mathfrak{B}$ is invariant under the action of $\mathcal{T}_{t}$ for all $t \geqslant 0$.

We will simply say that the semigroup is reduced by a normal operator $K$ when it is reduced by the algebra generated by $K$.

Remark 4. Suppose that $\mathcal{P}$ is a completely positive map defined on $\mathfrak{B}$ such that $\mathcal{P}(\mathbf{1})=1$. If $\mathcal{P}$ is reduced by an Abelian sub $C^{*}$-algebra $\mathfrak{A}$ of the algebra $\mathfrak{B}$, then its restriction $\mathbf{P}$ to $\mathfrak{A}$ defines a norm-continuous kernel. 
Indeed, since $\mathfrak{A}$ is an Abelian $C^{*}$-algebra which contains the unit, its set of characters $\sigma(\mathfrak{A})$ is a $w^{*}$-compact Hausdorff space (see [5], Theorem 2.1.11A, p. 62).

$\mathfrak{A}$ is isomorphic to the algebra of continuous functions $C(\sigma(\mathfrak{A}))$ via the Gelfand transform: $a \mapsto \hat{a}$, where $\hat{a}(\gamma)=\gamma(a)$, for all $a \in \mathfrak{A}, \gamma \in \sigma(\mathfrak{A})$.

Define $\mathbf{P} \hat{a}=\widehat{\mathcal{P}(a)}$. Then $\mathbf{P}: C(\sigma(\mathfrak{A})) \rightarrow C(\sigma(\mathfrak{A}))$ is linear, positive and continuous in norm since for all $a \in \mathfrak{A}$ :

$$
\|\mathbf{P} \hat{a}\|=\|\widehat{\mathcal{P}(a)}\| \leqslant\|a\|=\|\hat{a}\| .
$$

Therefore, by the disintegration of measures property (see [12]), there exists a kernel $P: \sigma(\mathfrak{A}) \times \mathcal{B}(\sigma(\mathfrak{A})) \rightarrow[0,1]$ such that $P(\psi, \cdot)$ is a (Radon) probability measure for all $\psi \in \sigma(\mathfrak{A}), P(\cdot, A)$ is a continuous function, for all $A \in \mathcal{B}(\mathfrak{A})$ and

$$
\mathbf{P} \hat{a}(\psi)=\int_{\sigma(\mathfrak{A})} P(\psi, \mathrm{d} \varphi) \varphi(a) .
$$

Remark 5. It follows immediately from the previous remark that if a Feller quantum Markov semigroup $\mathcal{T}$ (in particular, a norm-continuous semigroup) defined on $\mathfrak{B}$ is reduced by an Abelian $C^{*}$-subalgebra $\mathfrak{A}$ of $\mathfrak{B}$, there exists a classical Feller semigroup which is isomorphic to the restriction of $\mathcal{T}$ to $\mathfrak{A}$, called the reduced semigroup.

Given a *-Abelian subalgebra $\mathfrak{A} \subseteq \mathfrak{L}(\mathfrak{h})$, it is included in its commutant $\mathfrak{A}^{\prime}$ which is a von Neumann algebra. $\mathfrak{A}$ is maximal if $\mathfrak{A}=\mathfrak{A}^{\prime}$, and in that case $\mathfrak{A}$ becomes a von Neumann algebra too.

Remark 6. Suppose that $\mathcal{T}$ is a quantum Markov semigroup defined on $\mathfrak{L}(\mathfrak{h})$ which is reduced by a maximal Abelian von Neumann subalgebra $\mathfrak{A}$. Then there exists a compact Hausdorff space $E$ endowed with a Radon measure $\mu$ such that the restriction of $\mathcal{T}$ to $\mathfrak{A}$ is ${ }^{*}$-isomorphic to a classical Markov semigroup $\left(\mathbf{T}_{t}\right)_{t \in \mathbb{R}_{+}}$on $L^{\infty}(E, \mu)$. Moreover, if the semigroup $\mathcal{T}$ is quantum Feller, then $\left(\mathbf{T}_{t}\right)_{t \in \mathbb{R}_{+}}$is a Feller semigroup.

The above remark follows directly from the Spectral Theorem: Since $\mathfrak{A}$ is maximal Abelian, there exists a triple $(E, \mu, U)$, where $E$ is a compact second countable Hausdorff space, $\mu$ a Radon measure on $E$ and $U$ is an isometry from $L^{2}(E, \mu)$ onto $\mathfrak{h}$. $E$ is in fact the space of characters of $\mathfrak{A}$ which is $w^{*}$-compact.

So that $\mathcal{U}: L^{\infty}(E, \mu) \rightarrow \mathfrak{A}$, defined by $\mathcal{U}(f)=U M_{f} U^{*}$, where $f \in L^{\infty}(E, \mu)$ and $M_{f}$ denotes the multiplication operator by $f$ in $L^{2}(E, \mu)$, is an isometric *-isomorphism of algebras.

A semigroup $\left(\mathbf{T}_{t}\right)_{t \in \mathbb{R}_{+}}$is defined on $L^{\infty}(E, \mu)$ through the relation

$$
M_{\mathbf{T}_{t} f}=U^{*} \mathcal{T}_{t}\left(U M_{f} U^{*}\right) U
$$

for all $f \in L^{\infty}(E, \mu)$.

The semigroup $\left(\mathbf{T}_{t}\right)_{t \in \mathbb{R}_{+}}$preserves the identity, since $U$ is an isometry. Moreover, $\left\|\mathcal{T}_{t}(x)\right\| \leqslant\|x\|(x \in \mathfrak{M})$ implies that $\mathbf{T}_{t}$ is a contraction. Therefore, $\left(\mathbf{T}_{t}\right)_{t \in \mathbb{R}_{+}}$is a Markov semigroup on $L^{\infty}(E, \mu)$.

If $\mathcal{T}$ is Feller, this property is inherited by the classical reduction through Remark 5.

The previous results motivate the study of classical reductions of quantum Markov semigroups by Abelian algebras generated by a self-adjoint operator. To this end we refine below the applications of the Spectral Theorem to reduce completely positive maps. Given a normal operator $K$, we denote $W^{*}(K)$ its generated von Neumann algebra which coincides with the weak closure of the $C^{*}$-algebra $C^{*}(K)$ generated by the same operator.

The Abelian algebra $W^{*}(K)$ is maximal if and only if $K$ is multiplicity-free or non-degenerate. If $K$ is bounded, non-degeneracy means that there exists a cyclic vector for $C^{*}(K)$, that is, $\{f(K) \mathbf{w}: f \in C(\mathbf{S p}(K))\}$ is dense in $\mathfrak{h}$ for some vector $\mathbf{w}$, where $\operatorname{Sp}(K)$ denotes the spectrum of $K$. If $K$ is unbounded, it is non-degenerate if there is a vector $\mathbf{w}$ in the intersection of all domains $D\left(K^{n}\right)(n \geqslant 1)$, such that the subspace spanned by the vectors $\left(K^{n} \mathbf{w} ; n \geqslant 1\right)$ is dense in $\mathfrak{h}$. 
If $K$ is degenerate, one can decompose the Hilbert space in orthogonal subspaces on which $K$ is multiplicityfree. Here below we will deal with this more general case.

We denote $\xi$ the spectral measure of $K$. In addition, given a Radon measure on the measurable space $(\mathbf{S p}(K), \mathcal{B}(\mathbf{S p}(K)))$ we denote $L(\mathbf{S p}(K))$ the *-algebra which is obtained as the quotient of the set of Borel functions by null functions under the given Radon measure.

Lemma 1. Assume that $\mathcal{P}$ is a normal linear completely positive map defined on $\mathfrak{M}$ and such that $\mathcal{P}(\mathbf{1})=\mathbf{1}$. Let $K$ be an unbounded self-adjoint operator affiliated with $\mathfrak{M}$. Then the following propositions are equivalent:

(i) $W^{*}(K)$ is invariant under $\mathcal{P}$.

(ii) For any projection $p \in W^{*}(K), \mathcal{P}(p) \in W^{*}(K)$.

(iii) For all $A \in \mathcal{B}(\mathbf{S p}(K)), \mathcal{P}(\xi(A)) \in W^{*}(K)$.

(iv) There exists a kernel $P: \mathbf{S p}(K) \times \mathcal{B}(\mathbf{S p}(K)) \rightarrow \mathbb{R}^{+}$such that $P(x, \mathbf{S p}(K))=1$, for all $x \in \mathbf{S p}(K)$ and

$$
\mathcal{P}(\xi(A))=\int_{\mathbf{S p}(K)} \xi(\mathrm{d} x) P(x, A),
$$

for all $A \in \mathcal{B}(\mathbf{S p}(K))$.

Proof. Clearly, (i) implies (ii) which in turn implies (iii). The equivalence of (i) and (iii) follows from a straightforward application of the Spectral Theorem for general self-adjoint operators, since $\mathcal{P}$ is linear and normal. So that (i), (ii), and (iii) are equivalent.

To prove that (iii) implies (iv), we first notice that $\mathcal{P} \circ \xi$ is an operator valued measure. Indeed, since $\xi$ is the spectral measure of $K$ and $\mathcal{P}$ is linear and completely positive, the map $\mathcal{P} \circ \xi$ is additive on $\mathcal{B}(\mathbf{S p}(K))$. Moreover, take any pairwise disjoint sequence $\left(A_{n}\right)_{n \in \mathbb{N}}$ of Borel subsets of $\mathbf{S p}(K)$. The projection $\sum_{n} \xi\left(A_{n}\right)$ exists as a strong limit of the partial sums $\sum_{k \leqslant n} \xi\left(A_{k}\right)$. Moreover $\sum_{n} \xi\left(A_{n}\right)=1$.u.b. $\sum_{k \leqslant n} \xi\left(A_{k}\right)$ in the order of positive operators. Thus, the normality of the map $\mathcal{P}$ yields $\mathcal{P}\left(\sum_{n} \xi\left(A_{n}\right)\right)=$ l.u.b.P $\left(\sum_{k \leqslant n} \xi\left(A_{k}\right)\right)$, and $\mathcal{P} \circ \xi$ is an operator-valued measure.

If we assume (iii), given any $A \in \mathcal{B}(\mathbf{S p}(K))$, the Spectral Theorem implies that there exists $P(\cdot, A) \in L(\mathbf{S p}(K))$ such that

$$
\mathcal{P} \circ \xi(A)=\int_{\mathbf{S p}(K)} \xi(\mathrm{d} x) P(x, A) .
$$

Denote $\left(e_{n}\right)_{n \in \mathbb{N}}$ an orthonormal basis of $\mathfrak{h}$ and define the positive measure $\mu=\sum_{n} 2^{-n}\left\langle e_{n}, \xi(\cdot) e_{n}\right\rangle$. Since $\mathcal{P} \circ \xi$ is an operator-valued measure, it follows that $A \mapsto P(x, A)$ satisfies

$$
P\left(x, \bigcup_{n} A_{n}\right)=\sum_{n} P\left(x, A_{n}\right),
$$

for $\mu$-almost all $x \in \mathbf{S p}(K)$.

Since $\mu$ is a probability measure, it is tight on $\mathbf{S p}(K) \subseteq \mathbb{R}$. Therefore, for each $n \geqslant 1$, there exists a compact $K_{n}$ such that $\mu\left(K_{n}\right) \geqslant 1-2^{-n}$, so that $J=\bigcup_{n} K_{n} \subseteq \mathbf{S p}(K)$ satisfies $\mu(J)=1$. We imbed $J$ in $[-\infty, \infty]$, and consider $\mu$ as a probability measure defined on $[-\infty, \infty]$, supported by $J$. Let denote $\mathfrak{k}$ a vector space over the field of rational numbers, closed for lattice operations $\vee, \wedge$, dense in $C([-\infty, \infty])$ and such that $1 \in \mathfrak{k}$. Define

$$
A=\{x \in J: f \mapsto P(x, f) \text { is a positive } \mathbb{Q} \text {-linear form on } \mathfrak{k} \text { and } P(x, 1)=1\} .
$$

For all $x \in A, P(x, \cdot)$ can be extended as a positive linear form to all of $C([-\infty, \infty])$, and then to $L([-\infty, \infty])$. Moreover, $\mu(A)=1$ and

$$
\mu\left(\left\{x \in \mathbf{S p}(K): P\left(x, J^{c}\right)=0\right\}\right)=1 .
$$


We can complete the definition of the kernel $P$ choosing $P(x, \cdot)=\theta(\cdot)$ for all $x \notin A$, where $\theta$ is an arbitrary probability measure.

Finally to prove that (iv) implies (iii), it suffices to apply the Spectral Theorem again which yields $\int \xi(\mathrm{d} x) P(x, A) \in W^{*}(K)$.

Remark 7. It is worth noticing that if $K$ is degenerate, we have $W^{*}(K) \subset W^{*}(K)^{\prime}$ strictly and $W^{*}(K)^{\prime}$ is not Abelian. Indeed, an example borrowed to Pedersen [32] shows the first assertion. Suppose that $K u=\lambda u$ and $K v=\lambda v$ for two orthogonal unit vectors $u$ and $v$. There exists a unitary operator $W^{\prime}$ such that $W^{\prime} u=v, W^{\prime} v=-u$ and $W^{\prime}=\mathbf{1}$ on the orthogonal complement of $\mathbb{C} x \oplus \mathbb{C} y$. One can check easily that all elements $a \in W^{*}(K)$ have to satisfy $\langle(u+v), a(u-v)\rangle=0$, while $\left\langle(u+v), W^{\prime}(u-v)\right\rangle=2$. So that $W^{\prime} \notin W^{*}(K)$, however a direct computation shows that $W^{\prime} \in W^{*}(K)^{\prime}$. On the other hand, if one assume $W^{*}(K)^{\prime}$ to be commutative, then it has a separating vector. This means that the von Neumann algebra $W^{*}(K)$ has a cyclic vector and this property is equivalent to its maximality, that is $W^{*}(K)=W^{*}(K)^{\prime}$, contradicting the degeneracy of $K$.

The above discussion shows that the reduction of a completely positive map by the commutant $W^{*}(K)^{\prime}$ of the von Neumann algebra generated by $K$ leads to a classical kernel if and only if $K$ is non-degenerate.

\subsection{The $C^{*}$-case}

Consider first a semigroup $\mathcal{T}$ defined on a $C^{*}$-algebra $\mathfrak{B}$ and let a bounded normal operator $K$ be given. We then characterize the classical reduction of the semigroup as follows.

Theorem 1. Assume that $K$ is a normal operator in the $C^{*}$-algebra $\mathfrak{B}$ and call $C^{*}(K)$ the Abelian $C^{*}$-algebra generated by $K$. Then a norm-continuous quantum Markov semigroup $\mathcal{T}$ defined on $\mathfrak{B}$ is reduced by $C^{*}(K)$ if and only if $\mathcal{L}\left(K^{n}\right) \in C^{*}(K)$ for all $n \in \mathbb{N}$.

In particular, suppose that the generator is implemented by (4), where $H$ and $V$ satisfy:

(i) $[H, K] \in C^{*}(K)$;

(ii) $V^{*} V \in C^{*}(K)$;

(iii) For each $n \in \mathbb{N}$, there exists a constant $\alpha_{n} \in \mathbb{C}$ such that

$$
V K^{n}-\pi\left(K^{n}\right) V=\alpha_{n} V
$$

Then, the semigroup $\mathcal{T}$ is reduced by the algebra $C^{*}(K)$.

Proof. Let $\mathcal{K}$ denote the ${ }^{*}$-subalgebra generated by the commuting variables $\mathbf{1}, K, K^{*} . \mathcal{K}$ is strongly dense in $C^{*}(K)$. On the other hand, $\mathcal{T}$ is norm-continuous, so that $C^{*}(K)$ is invariant under $\mathcal{T}$ if and only if $\mathcal{L}(\mathcal{K}) \subseteq C^{*}(K)$. Since $\mathcal{L}(\mathbf{1})=0$ and $\mathcal{L}\left(K^{* n}\right)=\mathcal{L}\left(K^{n}\right)^{*}$, it follows easily that $\mathcal{L}(\mathcal{K}) \subseteq C^{*}(K)$ if and only if $\mathcal{L}\left(K^{n}\right) \in C^{*}(K)$, for all $n \in \mathbb{N}$.

To prove the second part of the theorem, from hypothesis (i) and the derivation property of $[H, \cdot]$ it follows that $\left[H, K^{n}\right] \in C^{*}(K)$ for all $n \in \mathbb{N}$. On the other hand, hypothesis (iii) yields

$$
V^{*} V K^{n}-V^{*} \pi\left(K^{n}\right) V=\alpha_{n} V^{*} V
$$

so that $V^{*} \pi\left(K^{n}\right) V$ belongs to $C^{*}(K)$ as well as $V^{*} V K^{n}$ and $K^{n} V^{*} V$, applying (ii). As a result, $\mathcal{L}\left(K^{n}\right) \in C^{*}(K)$ for all $n \in \mathbb{N}$, and the proof is complete. 


\subsection{Norm-continuous semigroups defined on von Neumann algebras}

Given a normal operator $K, W^{*}(K)$ reduces the norm-continuous quantum Markov semigroup $\mathcal{T}$ if and only if $\mathcal{L}(x) \in W^{*}(K)$ for all $x \in W^{*}(K)$. This follows immediately from the definition of the generator. Here below a modification of this elementary result in case of non-degeneracy.

Theorem 2. If a bounded self-adjoint operator $K \in \mathfrak{M}$ is non-degenerate, $W^{*}(K)$ reduces the norm-continuous quantum Markov semigroup $\mathcal{T}$ if and only if $\mathcal{L}(x)$ commutes with $K$ for any $x \in W^{*}(K)$.

In particular, suppose that

(i) $[H, K] \in W^{*}(K)$, and

(ii) $\left[L_{k}, K\right]=c_{k} L_{k}$, where $c_{k}=c_{k}^{*} \in W^{*}(K)$, for all $k \in \mathbb{N}$.

Then $W^{*}(K)$ reduces the semigroup $\mathcal{T}$.

Proof. If $K$ is non-degenerate, then $W^{*}(K)$ is maximal Abelian and coincides with its commutator $W^{*}(K)^{\prime}$. Thus, $\mathcal{L}\left(W^{*}(K)\right) \subseteq W^{*}(K)$ if and only if $\mathcal{L}(x)$ lies in $W^{*}(K)^{\prime}$ for any element $x \in W^{*}(K)^{\prime}$.

To prove the second part, consider an arbitrary $x \in W^{*}(K)^{\prime}$. To compute $[\mathcal{L}(x), K]$, we first observe that

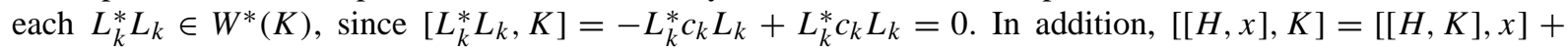
$[H,[x, K]]=0$. Therefore,

$$
\begin{aligned}
{[\mathcal{L}(x), K] } & =\left[\mathrm{i}[H, x]-\frac{1}{2} \sum_{k}\left[L_{k}^{*} L_{k} x-2 L_{k}^{*} x L_{k}+x L_{k}^{*} L_{k}, K\right]\right] \\
& =\mathrm{i}[[H, x], K 5]-\sum_{k}\left[L_{k}^{*} x L_{k}, K\right] \\
& =-\sum_{k}\left(-L_{k}^{*} c_{k} x L_{k}+L_{k}^{*}[x, K] L_{k}+L_{k}^{*} x c_{k} L_{k}\right) \quad\left(x, c_{k} \text { and } K \text { commute }\right) \\
& =0 .
\end{aligned}
$$

The previous theorem can be improved to consider an unbounded self-adjoint operator $K$ affiliated with the von Neumann algebra $\mathfrak{M}$.

For any quantum Markov semigroup $\mathcal{T}$ there exists $M>0$ and $\beta \in \mathbb{R}$ such that $\left\|\mathcal{T}_{t}\right\| \leqslant M \exp (\beta t)$ for all $t \geqslant 0$ (see [5], Proposition 3.1.6, p. 166). As a result, the resolvent $\mathcal{R}_{\lambda}(\cdot)$ of the semigroup is given by the Laplace transform

$$
\mathcal{R}_{\lambda}(x)=(\lambda \mathbf{1}-\mathcal{L})^{-1}(x)=\int_{0}^{\infty} \mathrm{d} t \mathrm{e}^{-\lambda t} \mathcal{T}_{t}(x),
$$

for all $x \in \mathfrak{M}$, whenever $\Re \lambda>\beta$.

Theorem 3. Let be $\mathcal{T}$ a quantum Markov semigroup on the von Neumann algebra $\mathfrak{M}$ and $K$ an unbounded selfadjoint operator affiliated with $\mathfrak{M}$. Then the following propositions are equivalent:

(i) The semigroup is reduced by $W^{*}(K)$.

(ii) For all $A \in \mathcal{B}(\mathbf{S p}(K))$ and any $t \geqslant 0, \mathcal{T}_{t}(\xi(A)) \in W^{*}(K)$.

(iii) The manifold $D(\mathcal{L}) \cap W^{*}(K)$ is non-trivial and for all $x \in D(\mathcal{L}) \cap W^{*}(K)$, it holds that $\mathcal{L}(x) \in W^{*}(K)$. 
(iv) There exists a classical Markov semigroup $\left(\mathbf{T}_{t}\right)_{t \in \mathbb{R}^{+}}$on $\mathbf{S p}(K)$ such that for all $f \in L(\mathbf{S p}(K))$,

$$
\mathcal{T}_{t}(f(K))=\int_{\mathbf{S p}(K)} \xi(\mathrm{d} x) \mathbf{T}_{t} f(x) .
$$

(v) For all $\lambda$ such that $\Re \lambda>\beta$ and all $A \in \mathcal{B}(\mathbf{S p}(K)) \mathcal{R}_{\lambda}(\xi(A)) \in W^{*}(K)$.

Proof. We clearly have the equivalence of (i), (ii) and (iii). Furthermore, the equivalence of (i) with (iv) follows from Lemma 1. Proposition (v) is equivalent to the existence of a family of kernels $R_{\lambda}$ on the spectrum of $K$ which defines a classical semigroup T. Thus (v) and (iv) are equivalent and this completes the proof.

The particular case of norm-continuous semigroups enjoys a richer characterization in terms of the generator.

Corollary 1. Suppose that $K$ is a non-degenerate self-adjoint operator. $W^{*}(K)$ reduces a norm-continuous quantum Markov semigroup $\mathcal{T}$ if and only if one of the following equivalent conditions is satisfied:

(i) $\mathcal{L}(\xi(A)) \in W^{*}(K)$ for all $A \in \mathcal{B}(\mathbf{S p}(K))$.

(ii) $[\mathcal{L}(\xi(A)), \xi(B)]=0$ for all $A, B \in \mathcal{B}(\mathbf{S p}(K))$.

(iii) There exists a dense domain $D \subseteq L(\mathbf{S p}(K))$ and an operator $\mathbf{L}: D \rightarrow L(\mathbf{S p}(K))$, such that for all $f \in D$, $f(K) \in D(\mathcal{L})$ and

$$
\mathcal{L}(f(K))=\int_{\mathbf{S p}(K)} \xi(\mathrm{d} x) \mathbf{L} f(x) .
$$

In particular, suppose that the generator $\mathcal{L}(\cdot)$ is given by (5) which in addition satisfies the two conditions below:

(a) $[H, \xi(A)] \in W^{*}(K)$, and

(b) $\left[L_{k}, \xi(A)\right]=c_{k}(A) L_{k}$, where $c_{k}(A)$ is a self-adjoint element in $W^{*}(K)$, for all $k \in \mathbb{N}$ and $A \in \mathcal{B}(\mathbf{S p}(K))$.

Then $W^{*}(K)$ reduces the semigroup $\mathcal{T}$.

Proof. The generator $\mathcal{L}(\cdot)$ is everywhere defined since the semigroup is norm-continuous. Thus, the equivalence of (i)-(iii) with (i) of the previous result is a simple consequence of the Spectral Theorem.

The last part follows from the first and Theorem 2 applied to $\xi(A)$.

\section{Semigroups with form-generators}

Finally, if the generator is given as a form through a Master Equation, for $\mathfrak{M}=\mathfrak{L}(\mathfrak{h})$, the above results have to be amended as follows.

Theorem 4. Assume that for all $x \in W^{*}(K)$, and all spectral projection $\xi(A)$, where $A \in \mathcal{B}(\mathbf{S p}(K))$ is such that $\xi(A)(D) \subseteq D$ it holds

$$
\mathfrak{L}(x)(v, \xi(A) u)=\mathfrak{L}(x)(\xi(A) v, u),
$$

for all $(u, v) \in D \times D$. Then the minimal semigroup $\mathcal{T}$ is reduced by $K$.

In particular, this is the case when the following two conditions hold: 
(a) The operator $G$ is affiliated with $W^{*}(K)$,

(b) Given any $x \in W^{*}(K),(u, v) \in D \times D, \ell \geqslant 1$,

$$
\left\langle L_{\ell} v, x L_{\ell} \xi(A) u\right\rangle=\left\langle L_{\ell} \xi(A) v, x L_{\ell} u\right\rangle,
$$

for all $A \in \mathcal{B}(\mathbf{S p}(K))$ such that $\xi(A)(D) \subseteq D$.

Proof. The proof follows the construction of the minimal quantum dynamical semigroup associated to the form $\mathfrak{E}(\cdot)$, as presented by Chebotarev (see [9]) and extensively used by him and Fagnola in their joint research on the Markov property of this minimal semigroup (see [8]).

Define $\mathcal{T}_{t}^{(0)}(x)=x$. Then, clearly $\left\langle v, \mathcal{T}_{t}^{(0)}(x) p u\right\rangle=\left\langle p v, \mathcal{T}_{t}^{(0)}(x) u\right\rangle$, for all $x \in W^{*}(K)$, all projection $p=\xi(A)$ leaving $D$ invariant, $(u, v) \in D \times D$. We follow by defining $\mathcal{T}_{t}^{(1)}(x)$ as follows: for each $(u, v) \in D \times D$,

$$
\left\langle v, \mathcal{T}_{t}^{(1)}(x) u\right\rangle=\langle v, x u\rangle+\int_{0}^{t} \mathfrak{L}\left(\mathcal{T}_{s}^{(0)}(x)\right)(v, u) \mathrm{d} s .
$$

Take $x \in W^{*}(K)$ a projection $p=\xi(A)$ as before, and apply hypothesis (24). Then it follows that

$$
\left\langle v, \mathcal{T}_{t}^{(1)}(x) p u\right\rangle=\left\langle p v, \mathcal{T}_{t}^{(1)}(x) u\right\rangle .
$$

This yields that $\mathcal{T}_{t}^{(1)}(x) \in W^{*}(K)$ if $x \in W^{*}(K)$.

By induction, suppose $\mathcal{T}_{t}^{(0)}(\cdot), \ldots, \mathcal{T}_{t}^{(n)}(\cdot)$ constructed and reduced by $W^{*}(K)$, then define $\mathcal{T}_{t}^{(n+1)}(\cdot)$ through the relation

$$
\left\langle v, \mathcal{T}_{t}^{(n+1)}(x) u\right\rangle=\langle v, x u\rangle+\int_{0}^{t} \mathfrak{E}\left(\mathcal{T}_{s}^{(n)}(x)\right)(v, u) \mathrm{d} s .
$$

By the induction hypothesis, and (24) again, it follows that

$$
\left\langle v, \mathcal{T}_{t}^{(n+1)}(x) p u\right\rangle=\left\langle p v, \mathcal{T}_{t}^{(n+1)}(x) u\right\rangle,
$$

for all projection $p=\xi(A)$ such that $p(D) \subseteq D$ and $(u, v) \in D \times D$, whenever $x \in W^{*}(K)$. Therefore, $K$ reduces the whole sequence $\left(\mathcal{T}^{(n)}\right)_{n \in \mathbb{N}}$. This sequence is used in the construction of the minimal quantum dynamical semigroup as follows. It is proved that $\left\langle u, \mathcal{T}_{t}^{(n)}(x) u\right\rangle$ is increasing with $n$ and $\left\langle u, \mathcal{T}_{t}(x) u\right\rangle$ is defined as its limit, for all $u \in \mathfrak{h}, x \in \mathfrak{L}(\mathfrak{h})$ (see [14]). Then by polarization $\left\langle v, \mathcal{T}_{t}(x) u\right\rangle$ is obtained. Thus, the minimal quantum dynamical semigroup $\mathcal{T}$ satisfies $\mathcal{T}_{t}(\mathbf{1}) \leqslant \mathbf{1}$, and given any other $\sigma$-weakly continuous family $\left(\mathcal{S}_{t}\right)_{t \in \mathbb{R}^{+}}$satisfying (8) and every positive operator $x \in \mathfrak{L}(\mathfrak{h})$, it holds $\mathcal{T}_{t}(x) \leqslant \mathcal{S}_{t}(x)$, for all $t \geqslant 0$. Moreover, since $\mathcal{T}_{t}^{(n)}\left(W^{*}(K)\right) \subseteq W^{*}(K)$, for all $n \in \mathbb{N}$ and $t \geqslant 0$, it follows that $K$ reduces the minimal quantum dynamical semigroup.

Assume now hypotheses (a) and (b). Condition (a) implies that $G \xi(A)=\xi(A) G$ for all projection $\xi(A)$ leaving $D$ invariant. Moreover, (b) yields $\sum_{\ell}\left\langle L_{\ell} v, x L_{\ell} \xi(A) u\right\rangle=\sum_{\ell}\left\langle L_{\ell} \xi(A) v, x L_{\ell} u\right\rangle$ and this, together with (a), clearly determine (24) and the proof is complete.

Corollary 2. With the notations and assumptions previous to the above theorem, suppose that in addition the two hypotheses below are satisfied:

(a) $G$ is affiliated with $W^{*}(K)$,

(b) For all $\ell \geqslant 1$ and any $A \in \mathcal{B}(\mathbf{S p}(K))$ such that $\xi(A)$ leaves $D$ invariant, there exists a selfadjoint operator $c_{\ell}(A) \in W^{*}(K)$, such that

$$
L_{\ell} \xi(A)=\left(\xi(A)+c_{\ell}(A)\right) L_{\ell} .
$$


Then $K$ reduces the minimal quantum dynamical semigroup $\mathcal{T}$.

Proof. Hypothesis (a) is identical to condition (a) of the previous theorem. On the other hand, if $x \in W^{*}(K)$, and $A \in \mathcal{B}(\mathbf{S p}(K))$ is such that $\xi(A)$ leaves $D$ invariant

$$
\begin{aligned}
\left\langle L_{\ell} \xi(A) v, x L_{\ell} u\right\rangle & =\left\langle\xi(A) L_{\ell} v, x L_{\ell} u\right\rangle+\left\langle c_{\ell}(A) L_{\ell} v, x L_{\ell} u\right\rangle \\
& =\left\langle L_{\ell} v, x \xi(A) L_{\ell} u\right\rangle+\left\langle L_{\ell} v, x c_{\ell}(A) L_{\ell} u\right\rangle \\
& =\left\langle L_{\ell} v, x\left(L_{\ell} \xi(A)-c_{\ell}(A) L_{\ell}\right) u\right\rangle+\left\langle L_{\ell} v, x c_{\ell}(A) L_{\ell} u\right\rangle \\
& =\left\langle L_{\ell} v, x L_{\ell} \xi(A) u\right\rangle,
\end{aligned}
$$

for all $(u, v) \in D \times D$. Thus, condition (b) of Theorem 4 is satisfied and the proof is complete.

\subsection{Returning to examples}

\subsubsection{The harmonic oscillator}

In Example 2.4.1 $\mathcal{L}(x)$ was indeed a form-generator which should be more rigorously written $\mathfrak{E}(x)$. The expression of the formal generator $\mathfrak{L}(\cdot)$ suggest to consider the reduction by $W^{*}(N)$. Indeed, $\mathbf{S p}(N)=\mathbb{N}$, the elements $e_{n}$ are the eigenvectors of $N$ and for any bounded function $f: \mathbb{N} \rightarrow \mathbb{C}$ a straightforward computation yields

$$
\mathfrak{L}(f(N))\left(v,\left|e_{n}\right\rangle\left\langle e_{n}\right| u\right)=\mathfrak{L}(f(N))\left(\left|e_{n}\right\rangle\left\langle e_{n}\right| v, u\right)=L f(n)\left\langle v, e_{n}\right\rangle\left\langle e_{n}, u\right\rangle,
$$

where,

$$
L f(n)=\lambda_{n}(f(n+1)-f(n))+\mu_{n}(f(n-1)-f(n)),
$$

and

$$
\lambda_{n}=A v(n+1), \quad \mu_{n}=A(v+1) n \quad(n \in \mathbb{N}) .
$$

As it is easily seen, the expression (27) corresponds to the generator of a classical birth and death Markov semigroup, with birth rate $\lambda_{n}$ and death rate $\mu_{n}$.

\subsubsection{The quantum Brownian motion}

The commutative von Neumann subalgebra $W^{*}(q)$ of $\mathfrak{M}$ whose elements are multiplication operators $M_{f}$ by a function $f \in L^{\infty}\left(\mathbb{R}^{d} ; \mathbb{C}\right)$ is $\mathcal{T}$-invariant and $\mathcal{T}_{t}\left(M_{f}\right)=M_{T_{t} f}$ where

$$
\left(T_{t} f\right)(x)=\frac{1}{(2 \pi t)^{d / 2}} \int_{\mathbb{R}^{d}} f(y) \mathrm{e}^{-|x-y|^{2} / 2 t} \mathrm{~d} y .
$$

The same conclusion holds for the commutative algebra $W^{*}(p)=F^{*} W^{*}(q) F$, where $F$ denotes the Fourier transform (see [18]). Therefore, this QMS named quantum Brownian motion semigroup contains a couple of noncommuting classical Brownian semigroups as classical reductions.

Moreover, notice that the von Neumann algebra $W^{*}(N)$ generated by the number operator $N=\sum_{j} a_{j}^{\dagger} a_{j}$ is also $\mathcal{T}$ invariant and the classical semigroup obtained by restriction of $\mathcal{T}$ to $W^{*}(N)$ like in the previous example is a birth and death on $\mathbb{N}$ with birth rates $(n+1)_{n \geqslant 0}$ and death rates $(n)_{n} \geqslant 0$.

\subsubsection{The quantum exclusion semigroup}

Given $\eta \in \mathbf{S}, i, j \in \mathbb{N}$, define $c_{i, j}(\eta)=\eta(i)(1-\eta(j)) \gamma_{i, j}$. 
Proposition 1. For each $x \in \mathfrak{A}\left(\mathfrak{h}_{0}\right)$ the unbounded operator

$$
\mathcal{L}(x)=\mathrm{i}[H, x]-\frac{1}{2} \sum_{i, j}\left(L_{i, j}^{*} L_{i, j} x-2 L_{i, j}^{*} x L_{i, j}+x L_{i, j}^{*} L_{i, j}\right),
$$

whose domain contains the dense manifold $\mathfrak{v}$, is the generator of a quantum Feller semigroup $\mathcal{T}$ on the $C^{*}$-algebra $\mathfrak{A}\left(\mathfrak{h}_{0}\right)$. This semigroup is extended into a $\sigma$-weak continuous $Q M S$ defined on the whole algebra $\mathfrak{L}(\mathfrak{h})$.

Moreover, the semigroup is reduced by the algebra $W^{*}(H)$. The reduced semigroup $\mathbf{T}$ corresponds to a classical exclusion process (see [24]) with generator

$$
L f(\eta)=\sum_{i, j} c_{i, j}(\eta)\left(f\left(\eta+1_{j}-1_{i}\right)-f(\eta)\right)
$$

for all bounded cylindrical function $f: \mathbf{S} \rightarrow \mathbb{R}$.

Proof. We first notice that there exists the minimal quantum Markov semigroup associated with the generator (30). Indeed this holds since $\mathfrak{v}$ is dense in $\mathfrak{h}=\Gamma_{f}\left(\mathfrak{h}_{0}\right)$, and it is a core for $G=-\mathrm{i} H-\frac{1}{2} \sum_{i, j} L_{i, j}{ }^{*} L_{i, j}$, which is the generator of a contraction semigroup, and $\mathfrak{v}$ is also contained in the domain of all the operators $L_{i, j}$, and the Markovian property is guaranteed by $\mathcal{L}(\mathbf{1})=0$. Let denote $\mathcal{T}$ this minimal semigroup (see [11] for further details) which is defined through the use of the $\sigma$-weak topology in all of $\mathfrak{L}(\mathfrak{h})$. It satisfies the equation

$$
\left\langle v, \mathcal{T}_{t}(x) u\right\rangle=\langle v, x u\rangle+\int_{0}^{t}\left\langle v, \mathcal{L}\left(\mathcal{T}_{s}(x)\right) u\right\rangle \mathrm{d} s,
$$

where $u, v \in \mathfrak{v}, x \in \mathfrak{L}(\mathfrak{h})$.

Take $x \in \mathfrak{A}\left(\mathfrak{h}_{0}\right)$ and $\Lambda \in \mathfrak{P}_{f}(\mathbb{N})$. Call $E_{\Lambda}$ the projection of $\mathfrak{h}=\Gamma_{f}\left(\mathfrak{h}_{0}\right)$ onto $\mathfrak{h}^{\Lambda}=\Gamma_{f}\left(\mathfrak{h}_{0}^{\Lambda}\right)$. Notice that for all $a \in \mathfrak{A}\left(\mathfrak{h}_{0}\right)$, the net of projected operators $E_{\Lambda} a E_{\Lambda} \in \mathfrak{A}\left(\mathfrak{h}_{0}^{\Lambda}\right)$ converges strongly to $a$, as $\Lambda \uparrow \mathbb{N}$ since $\mathfrak{A}\left(\mathfrak{h}_{0}\right)$ is the strong closure of $\mathfrak{D}=\bigcup \mathfrak{A}\left(\mathfrak{h}_{0}^{\Lambda}\right)$. Call $\mathbf{E}_{\Lambda}(a)=E_{\Lambda} a E_{\Lambda}$ the projection of an element of the algebra $\mathfrak{A}\left(\mathfrak{h}_{0}\right)$ and $\mathcal{T}_{t}^{\Lambda}(x)=E_{\Lambda} \mathcal{T}_{t}(x) E_{\Lambda}, x \in \mathfrak{A}\left(\mathfrak{h}_{0}^{\Lambda}\right), t \geqslant 0$. This is a semigroup acting on $\mathfrak{A}\left(\mathfrak{h}_{0}^{\Lambda}\right)$ whose generator, determined by (32), is $\mathcal{L}^{\Lambda}(x)=E_{\Lambda} \mathcal{L}(x) E_{\Lambda}$, for each $x \in \mathfrak{A}\left(\mathfrak{h}_{0}^{\Lambda}\right) . \mathcal{L}^{\Lambda}(x)$ is a matrix in a finite-dimensional space, so that it is a bounded operator. As a result, each $\mathcal{T}^{\Lambda}$ is a norm-continuous semigroup.

To prove that the minimal semigroup $\mathcal{T}$ satisfies the Feller property (M5F) on the algebra $\mathfrak{A}\left(\mathfrak{h}_{0}\right)$, we first consider $x \in \mathfrak{D}$. So that there is $\Lambda_{0} \in \mathfrak{P}_{f}(\mathbb{N})$ such that $x \in \mathfrak{A}\left(\mathfrak{h}_{0}^{\Lambda}\right)$, which yields $\mathbf{E}_{\Lambda}(x)=x$ for all $\Lambda \in \mathfrak{P}_{f}(\mathbb{N})$ containing $\Lambda_{0}$. Then, for all such $\Lambda$,

$$
\left\|\mathcal{T}_{t}(x)-x\right\| \leqslant\left\|\mathcal{T}_{t}(x)-\mathcal{T}_{t}^{\Lambda}(x)\right\|+\left\|\mathcal{T}_{t}^{\Lambda}(x)-x\right\| .
$$

Since $\mathbf{E}_{\Lambda} \circ \mathcal{T}_{t}(x)$ strongly converges to $\mathcal{T}_{t}(x)$ as $\Lambda$ increases, given any $\epsilon>0$ we can choose $\Lambda \in \mathfrak{P}_{f}(\mathbb{N})$ to have the first right-hand term in the previous inequality less than $\epsilon / 2$. On the other hand, for this $\Lambda$ we also have $\lim _{t \rightarrow 0}\left\|\mathcal{T}_{t}^{\Lambda}-\mathbf{1}\right\|=0$ and $t_{0}$ may be selected to have the second right-hand term in the inequality less than $\epsilon / 2$ too for any $t<t_{0}$. This proves (M5F) for $x \in \mathfrak{D}$.

If $x \in \mathfrak{A}\left(\mathfrak{h}_{0}\right)$, we pick a net $x_{\Lambda} \in \mathfrak{A}\left(\mathfrak{h}_{0}^{\Lambda}\right)$ which strongly converges to $x$, use the fact that $\mathcal{T}_{t}(\cdot)$ is a contraction and the property (M5F) proved for elements in $\mathfrak{D}$ to conclude. $\mathcal{T}$ is thus a quantum Feller semigroup on the algebra $\mathfrak{A}\left(\mathfrak{h}_{0}\right)$.

To study the classical reduction, it suffices to use the C.A.R. Indeed, $\left[b_{k}^{\dagger} b_{k}, b_{j}^{\dagger} b_{i}\right]=\left(\delta_{k j}-\delta_{k i}\right) b_{j}^{\dagger} b_{i}$. Moreover, since each operator $L_{i, j}$ is bounded and $L_{i, j} \mathfrak{v} \subset \mathfrak{v} \subset D(H)$, the commutator $\left[H, L_{i, j}\right]$ is well defined on $\mathfrak{v}$ and can be extended to all of $\mathfrak{h}$ as a bounded operator since

$$
\left[H, L_{i, j}\right]=\sum_{k} E_{k} \sqrt{\gamma_{i, j}}\left[b_{k}^{\dagger} b_{k}, b_{j}^{\dagger} b_{i}\right]=\left(E_{j}-E_{i}\right) L_{i, j} .
$$


So that, adapting to this case the proof of Theorem 2, we conclude that $H$ reduces the semigroup.

We now obtain the expression of the reduced generator. The spectral decomposition of $H$ may be written,

$$
H=\sum_{\eta} E(\eta)|\eta\rangle\langle\eta|
$$

where $E(\eta)=\sum_{i} \eta(i) E_{i}$, for all configuration $\eta$. The algebra $\mathbf{C y l}(H)$ of operators of the form

$$
x=\sum_{\eta} f(\eta)|\eta\rangle\langle\eta|
$$

where $f: \mathbf{S} \rightarrow \mathbb{C}$ is a bounded cylindrical function, form a dense subalgebra of $C^{*}(H)$.

We compute $\mathcal{L}(x)$ for $x \in \mathbf{C y l}(H)$.

The following additional notation will be used: $i \stackrel{\eta}{\rightarrow} j$, means that $\eta(i)=1$ and $\eta(j)=0$ (under the configuration $\eta$ a particle occupying the site $i$ can move to the free site $j$ ).

An elementary computation yields

$$
\begin{aligned}
& L_{i, j}^{*} L_{i, j}|\eta\rangle\left\langle\eta\left|=\gamma_{i, j} \eta(i)(1-\eta(j))\right| \eta\right\rangle\langle\eta|, \\
& |\eta\rangle\left\langle\eta\left|L_{i, j}^{*} L_{i, j}=\gamma_{i, j} \eta(i)(1-\eta(j))\right| \eta\right\rangle\langle\eta|, \\
& L_{i, j}^{*}|\eta\rangle\left\langle\eta\left|L_{i, j}=\gamma_{i, j}(1-\eta(i)) \eta(j)\right| \eta-1_{j}+1_{i}\right\rangle\left\langle\eta-1_{j}+1_{i}\right| .
\end{aligned}
$$

From this it follows that

$$
\begin{aligned}
\mathcal{L}(|\eta\rangle\langle\eta|) & =-\frac{1}{2} \sum_{i, j}\left(L_{i, j}^{*} L_{i, j}|\eta\rangle\left\langle\eta\left|-2 L_{i, j}^{*}\right| \eta\right\rangle\left\langle\eta\left|L_{i, j}+\right| \eta\right\rangle\langle\eta| L_{i, j}^{*} L_{i, j}\right) \\
& =\sum_{i, j: j \stackrel{\eta}{\rightarrow} i} \gamma_{i, j}\left|\eta-1_{j}+1_{i}\right\rangle\left\langle\eta-1_{j}+1_{i}\left|-\sum_{i, j: i \stackrel{\eta}{\rightarrow} j} \gamma_{i, j}\right| \eta\right\rangle\langle\eta| .
\end{aligned}
$$

Now, for any $x=\sum_{\eta} f(\eta)|\eta\rangle\langle\eta| \in \mathbf{C y l}(H)$,

$$
\mathcal{L}(x)=\sum_{\eta}\left(\sum_{i, j: j \rightarrow i} \gamma_{i, j} f(\eta)\left|\eta-1_{j}+1_{i}\right\rangle\left\langle\eta-1_{j}+1_{i}\left|-\sum_{i, j} \gamma_{i, j} f(\eta)\right| \eta\right\rangle\langle\eta|\right),
$$

and notice that a change of variables $\eta \mapsto \eta-1_{j}+1_{i}$ yields

$$
\sum_{\eta} \sum_{i, j: j \rightarrow i} \gamma_{i, j} f(\eta)\left|\eta-1_{j}+1_{i}\right\rangle\left\langle\eta-1_{j}+1_{i}\left|=\sum_{\eta} \sum_{i, j: i \rightarrow j} \gamma_{i, j} f\left(\eta-1_{i}+1_{j}\right)\right| \eta\right\rangle\langle\eta| .
$$

Therefore, we finally obtain

$$
\mathcal{L}(x)=\sum_{\eta} \sum_{i, j: i \stackrel{\eta}{\rightarrow} j} \gamma_{i, j}\left(f\left(\eta-1_{i}+1_{j}\right)-f(\eta)\right)|\eta\rangle\langle\eta|,
$$

from which (31) follows.

The above expression gives the generator of the semigroup restricted to $W^{*}(H)$. $C^{*}(H)$ is isomorphic with the algebra $C(\mathbf{S p}(K))$ of continuous complex valued functions on the compact set $\mathbf{S p}(H)$ and contains continuous cylindrical functions as a uniformly dense sub-algebra. It is clear that $L$ given by (31) leaves the above dense subalgebra invariant, thus the reduced semigroup applies $C(\mathbf{S p}(H)$ into itself, moreover, the Feller continuity property is inherited from the quantum Markov semigroup. 


\section{Decoherence as a limit behavior}

Let be given a quantum Markov semigroup $\mathcal{T}$ defined on $\mathfrak{M}=\mathfrak{L}(\mathfrak{h})$. We introduce two additional notations:

$$
\mathfrak{F}(\mathcal{T})=\left\{x \in \mathfrak{M}: \mathcal{T}_{t}(x)=x, \text { for all } t \geqslant 0\right\}
$$

and

$$
\mathfrak{N}(\mathcal{T})=\left\{x \in \mathfrak{M}: \mathcal{T}_{t}\left(x^{*} x\right)=\mathcal{T}_{t}\left(x^{*}\right) \mathcal{T}_{t}(x), \text { for all } t \geqslant 0\right\} .
$$

The first set corresponds to invariant elements under the action of the semigroup, whereas the second consists of elements for which equality holds in Schwartz inequality for completely positive maps, a feature which is characteristic of an automorphism group. In general, $\mathfrak{F}(\mathcal{T}) \subseteq \mathfrak{N}(\mathcal{T})$. Assume further that a faithful normal stationary state $\omega_{\infty}=\operatorname{tr}\left(\rho_{\infty} \cdot\right)$ exists for the semigroup $\mathcal{T}$. Under this hypothesis, Frigerio and Verri proved in [19] that for a norm-continuous semigroup, $\mathfrak{F}(\mathcal{T}), \mathfrak{N}(\mathcal{T})$ are von Neumann algebras, the conditional expectations $\mathbb{E}^{\mathfrak{F}(\mathcal{T})}(\cdot)$ and $\mathbb{E}^{\mathfrak{N}(\mathcal{T})}(\cdot)$ exist and any other stationary state can be represented as $\omega=\omega_{\infty} \circ \mathbb{E}^{\mathfrak{F}(\mathcal{T})}(\cdot)$. In addition they proved that if $\mathfrak{F}(\mathcal{T})=\mathfrak{N}(\mathcal{T})$, then for any initial state $\omega$ and any element $x$ of the algebra, $\mathcal{T}_{* t}(\omega)(x)=\omega\left(\mathcal{T}_{t}(x)\right) \rightarrow \omega\left(\mathbb{E}^{\mathfrak{F}(\mathcal{T})}(x)\right)=\omega_{\infty}(x)$ as $t \rightarrow \infty$. Throughout the remains of the paper a semigroup is said to be weakly ergodic (or simply ergodic) when the above property holds. Since we are assuming all states to be normal, this means that for any initial density matrix $\rho, \mathcal{T}_{* t}(\rho)$ weakly converges to the invariant faithful density matrix $\rho_{\infty}$ associated to $\omega_{\infty}$ as $t \rightarrow \infty$. This result has been extended by Fagnola and Rebolledo in [15] to a general class of QMS. Moreover, for a generator given by a form like (7), with $G$ as in (6), where $H$ is a self-adjoint operator with pure point spectrum, one obtains the following nice characterization of weak ergodicity.

Theorem 5 (Fagnola-Rebolledo, Theorem II.2, [15]). Suppose that the minimal semigroup $\mathcal{T}$ associated to $\mathfrak{L}(\cdot)$ is Markov and that it has a normal faithful stationary state $\omega_{\infty}$.

Assume in addition that $H$ is a self-adjoint operator with pure point spectrum and either

(a) $H$ is bounded;

or

(b) $H$ is selfadjoint and $\mathrm{e}^{\mathrm{i} t H}(D) \subseteq D(G)$, where $D \subseteq D(G)$ is a dense linear subspace.

$$
\text { if }
$$

Let $\omega$ denote an arbitrary normal state. Then $\mathcal{T}_{t *}(\omega)$ converges in the $w^{*}$-topology to $\omega_{\infty}$ as $t \rightarrow \infty$ if and only

$$
\left\{L_{k}, L_{k}^{*}, H ; k \geqslant 1\right\}^{\prime}=\left\{L_{k}, L_{k}^{*} ; k \geqslant 1\right\}^{\prime}
$$

It is worth mentioning that sufficient conditions for the existence of a $\mathcal{T}$-stationary normal faithful state have been obtained in terms of the generator too in [16] and [17]. On the other hand, no stationary state exist for a transient quantum Markov semigroup. Transience and recurrence of quantum Markov semigroups have been studied in [18].

We now proceed with the definition of decoherence in our framework. To keep this notion close to the first approach of physicists, throughout this section we consider a self-adjoint operator $K$ with pure point spectrum and denote $\left(e_{n}\right)_{n \in \mathbb{N}}$ an orthonormal basis of eigenvectors of $K$.

Definition 4. We say that $K$ induces decoherence of the quantum Markov semigroup $\mathcal{T}$ if there exists a faithful $\mathcal{T}$-stationary density matrix which commutes with $K$.

Equivalently, $K$ induces decoherence of $\mathcal{T}$ if there exists a common faithful stationary density matrix for both $\mathcal{T}$ and the group $\alpha$ of automorphisms associated to $K, \alpha_{t}(x)=\exp (\mathrm{i} t K) x \exp (-\mathrm{i} t K),(x \in \mathfrak{M}, t \in \mathbb{R})$. 
Remark 8. In an interesting article (see [26]), Majewski and Streater introduced what they called the balance condition II. Their definition, applied to the dynamics $\alpha$ and $\mathcal{T}$ before, allows to easily infer a sufficient condition for decoherence as we show below.

Proposition 2. Assume $K$ to be a self-adjoint operator with pure-point spectrum and let $\alpha$ denote the automorphism group it induces. Suppose that there exists a normal faithful state $\omega$ such that the following balance equation (balance condition II in [26]) is satisfied,

$$
\omega\left(\alpha_{t}\left(a^{*}\right) b\right)=\omega\left(a^{*} \mathcal{T}_{t}(b)\right)
$$

for all $a, b \in \mathfrak{M}$. Then $\omega$ is a stationary state for both $\alpha$ and $\mathcal{T}$, and $K$ induces decoherence of the semigroup $\mathcal{T}$.

Proof. To derive the $\alpha$-invariance of $\omega$, choose first $b=\mathbf{1}$ in (34) and use the Markov property of $\mathcal{T}$. Similarly, taking $a=\mathbf{1}$ and letting $b$ in (34) arbitrary, yields the $\mathcal{T}$-invariance of $\omega$. Moreover, since $\omega$ is normal, there exists a density matrix $\rho$ which commutes with $K$ and such that $\omega(\cdot)=\operatorname{tr}(\rho \cdot)$. Thus $K$ induces decoherence of $\mathcal{T}$.

In concrete physical models though, condition (34) is oftenly hard to verify. Our aim in the remaining of the paper, is to look for applicable criteria on decoherence based on the structure of the semigroup generator $\mathcal{L}$. We start by some remarks and straightforward consequences of Definition 4.

Remark 9. Notice that if $K$ is non-degenerate, Definition 4 implies that for all density matrix $\rho$ there exists a sequence $\left(t_{r}\right)_{r \in \mathbb{N}}$ such that $t_{r} \rightarrow \infty$ and for all $n \neq m$,

$$
\frac{1}{t_{r}} \int_{0}^{t_{r}}\left\langle e_{m}, \mathcal{T}_{* s}(\rho) e_{n}\right\rangle \mathrm{d} s \rightarrow 0 .
$$

Moreover, we have the following easy proposition improving the above remark.

Proposition 3. Assume that a non-degenerate self-adjoint operator $K$ with pure point spectrum as before induces decoherence of $\mathcal{T}$ and that the semigroup is ergodic. Then, given any density matrix $\rho \in \mathfrak{I}_{1}(\mathfrak{h})$, and $n \neq m$, it holds

$$
\left\langle e_{m}, \mathcal{T}_{* t}(\rho) e_{n}\right\rangle \rightarrow 0
$$

as $t \rightarrow \infty$.

Proof. Since the semigroup is ergodic and $K$ induces decoherence, it holds that $\operatorname{tr}\left(\mathcal{T}_{* t}(\rho) x\right)$ converges to $\operatorname{tr}\left(\rho_{\infty} x\right)$ for all $x \in \mathfrak{M}$, as $t \rightarrow \infty$ and any density matrix $\rho$, where $\rho_{\infty}$ is a faithful density matrix which commutes with $K$.

The last statement follows straightforward from the above since $\left\langle e_{m}, \rho_{\infty} e_{n}\right\rangle=0$ for $n \neq m$.

Proposition 4. Suppose that the self-adjoint operator $K$ is non-degenerate and that $W^{*}(K)$ reduces the quantum Markov semigroup $\mathcal{T}$. If $K$ induces decoherence of $\mathcal{T}$, then the reduced semigroup has a faithful stationary probability measure.

Proof. Call $\rho$ a faithful stationary density matrix which commutes with $K$. So that $\rho$ can be written as

$$
\rho=\sum_{n} p(n)\left|e_{n}\right\rangle\left\langle e_{n}\right|
$$

where $\sum_{n} p(n)=\operatorname{tr}\left(\rho_{\infty}\right)=1$, and each $p(n)>0$ due to the faithfulness of $\rho$. Given any bounded function $f$ on the spectrum of $K$, it holds:

$$
\operatorname{tr}\left(\rho \mathcal{T}_{t}(f(K))\right)=\sum_{n} p(n) T_{t} f(n)=\operatorname{tr}(\rho f(K))=\sum_{n} p(n) f(n),
$$


for all $t \geqslant 0$. Thus, the density $p(n)$ defines a probability measure on $\mathbf{S p}(K)$ which is stationary under the reduced semigroup $\left(T_{t}\right)_{t \in \mathbb{R}^{+}}$.

It is an important problem for applications to Physics to know whether the knowledge of a stationary probability for a reduced semigroup leads to decoherence. To give a partial answer for a wide class of semigroups, we previously need to take care of some technical matters concerning the domain of the generator. Suppose that we are given a form-generator $\mathfrak{E}(\cdot)$ with which we construct the associated minimal quantum Markov semigroup (see $[11,8])$. We will assume here that the orthonormal basis of eigenvectors of $K$ can be chosen on the dense subset $D$ which is a core for the operator $G$ and all the operators $L_{k}$ defining $\mathfrak{E}(\cdot)$. Then, as proved in [11], Sections 2 and 3 , the linear space spanned by all the projections $\left|e_{n}\right\rangle\left\langle e_{m}\right|,(n, m \in \mathbb{N})$, is a core for the predual generator $\mathcal{L}_{*}$. As a result, all the operators $\mathcal{L}_{*}\left(\left|e_{n}\right\rangle\left\langle e_{n}\right|\right)$ are well defined.

Theorem 6. Suppose that the self-adjoint operator $K$ is non-degenerate and that $W^{*}(K)$ reduces the quantum Markov semigroup $\mathcal{T}$. Assume that there exists a faithful probability density $(p(\lambda))_{\lambda \in \mathbf{S p}(K)}$ on the spectrum of $K$ which is stationary for the reduced semigroup. If for all $n \in \mathbb{N}, \mathcal{L}_{*}\left(\left|e_{n}\right\rangle\left\langle e_{n}\right|\right)$ commutes with $K$, then $K$ induces decoherence of $\mathcal{T}$.

Proof. Call $\rho=p(K)=\sum_{n} p\left(\lambda_{n}\right)\left|e_{n}\right\rangle\left\langle e_{n}\right|$. We will prove that $\rho \in D\left(\mathcal{L}_{*}\right)$ and that $\mathcal{L}_{*}(\rho)=0$. Given any bounded function $f$ on $\mathbf{S p}(K)$, call $L$ the reduction of the generator, that is $\left\langle e_{n}, \mathcal{L}(f(K)) e_{n}\right\rangle=L f\left(\lambda_{n}\right)$. The hypothesis on the stationarity of $p$ is then expressed as $\sum_{n} p\left(\lambda_{n}\right) L f\left(\lambda_{n}\right)=0$.

Define

$$
\rho_{N}=\sum_{n \leqslant N} p\left(\lambda_{n}\right)\left|e_{n}\right\rangle\left\langle e_{n}\right| \quad(N \in \mathbb{N}) .
$$

Notice that $\rho_{N} \in D\left(\mathcal{L}_{*}\right)$, since each projection $\left|e_{n}\right\rangle\left\langle e_{n}\right|$ belongs to $D\left(\mathcal{L}_{*}\right)$. Moreover, $\mathcal{L}_{*}\left(\rho_{N}\right)$ is a trace-class operator as well (see Subsection 2.3). Since $\mathcal{L}_{*}\left(\left|e_{n}\right\rangle\left\langle e_{n}\right|\right)$ commutes with $K$ and $K$ is non-degenerate, $\mathcal{L}_{*}\left(\rho_{n}\right) \in$ $W^{*}(K)$ too.

Taking $N, M \in \mathbb{N}$, with $N>M$ say, one obtains

$$
\left|\operatorname{tr}\left(\left(\mathcal{L}_{*}\left(\rho_{N}\right)-\mathcal{L}_{*}\left(\rho_{M}\right)\right) x\right)\right| \leqslant C(x) \sum_{n=M+1}^{N} p\left(\lambda_{n}\right),
$$

for any fixed $x \in D(\mathcal{L})$, for a constant $C(x)>0$. Since $\sum_{n} p\left(\lambda_{n}\right)=1$, we obtain that $\mathcal{L}_{*}\left(\rho_{N}\right)$ weakly converges as $N \rightarrow \infty$. On the other hand, $\rho_{N}$ converges in the norm of the trace to $\rho$. Since $\mathcal{L}_{*}(\cdot)$ is weakly closed, then $\mathcal{L}_{*}(\rho)=\lim _{N} \mathcal{L}_{*}\left(\rho_{N}\right)$ and $\rho \in D\left(\mathcal{L}_{*}\right)$. Moreover, $\mathcal{L}_{*}(\rho)$ is a trace-class operator which commutes with $K$.

After the previous result, to prove that $\mathcal{L}_{*}(\rho)=0$ it suffices to show that $\left\langle e_{k}, \mathcal{L}_{*}(\rho) e_{k}\right\rangle=\operatorname{tr}\left(\mathcal{L}_{*}(\rho)\left|e_{k}\right\rangle\left\langle e_{k}\right|\right)=0$ for all $k \in \mathbb{N}$. Now,

$$
\begin{aligned}
\operatorname{tr}\left(\mathcal{L}_{*}(\rho)\left|e_{k}\right\rangle\left\langle e_{k}\right|\right) & =\operatorname{tr}\left(\rho \mathcal{L}\left(\left|e_{k}\right\rangle\left\langle e_{k}\right|\right)\right) \\
& =\sum_{n} p\left(\lambda_{n}\right)\left\langle e_{n}, \mathcal{L}\left(\left|e_{k}\right\rangle\left\langle e_{k}\right|\right) e_{n}\right\rangle \\
& =\sum_{n} p\left(\lambda_{n}\right) L 1_{\left\{\lambda_{k}\right\}}\left(\lambda_{n}\right) \\
& =0 \quad \text { (since } p \text { is stationary for the reduced semigroup) }
\end{aligned}
$$

Thus, $\mathcal{L}_{*}(\rho)=0$ and $\rho$ is a stationary state for $\mathcal{T}$. As a result, $K$ induces decoherence of the quantum Markov semigroup. 
Corollary 3. Suppose that the semigroup $\mathcal{T}$ is norm-continuous with generator given by (5) and satisfies the following hypotheses:

(i) $H$ has a pure point spectrum;

(ii) $\left[H, L_{k}\right]=\alpha_{k} L_{k}, \alpha_{k} \in \mathbb{R}$, for all $k \geqslant 1$;

(iii) the generalized commutants $\left\{H, L_{k}, L_{k}^{*}, k \in \mathbb{N}\right\}^{\prime}$ and $\left\{L_{k}, L_{k}^{*}, k \in \mathbb{N}\right\}^{\prime}$ coincide;

(iv) There is a faithful probability $p$ on the spectrum of $H$ solving the equation

$$
L_{k} p(H) L_{k}^{*}=L_{k}^{*} L_{k} p(H)=p(H) L_{k}^{*} L_{k} \quad(k \in \mathbb{N}) .
$$

Then the semigroup is reduced by $H, p(H)$ defines a stationary state for the semigroup and $H$ induces decoherence of $\mathcal{T}$. Given any density matrix $\rho, \mathcal{T}_{* t}(\rho) \rightarrow p(H)$ in the $w^{*}$-topology. As a result,

$$
\left\langle e_{n}, \mathcal{T}_{* t}(\rho) e_{m}\right\rangle \rightarrow 0
$$

as $t \rightarrow \infty$, for all $n \neq m, n, m \in \mathbb{N}$, where $\left(e_{n}\right)_{n \in \mathbb{N}}$ is an orthonormal basis of eigenvectors of $H$.

Proof. Hypothesis (ii) implies that $H$ reduces the semigroup. Then (iv) determines the existence of a faithful and normal stationary state given by a density matrix $\rho_{\infty}=p(H)$, since $\mathcal{L}_{*}(p(H))=0$. Thus $H$ induces decoherence of $\mathcal{T}$. Condition (iii) implies the convergence of $\mathcal{T}_{* t}(\rho)$ to $\rho_{\infty}$ for any density matrix $\rho$ by Theorem II.2 in [15] which has been recalled here in Theorem 5. Finally Proposition 3 leads to the conclusion.

The above result can be improved to consider more general QMS as follows

Corollary 4. Let $K$ be as in Theorem 6 . Assume that the semigroup $\mathcal{T}$ is reduced by $W^{*}(K)$ and that $\mathcal{L}_{*}\left(\left|e_{n}\right\rangle\left\langle e_{n}\right|\right) \in$ $W^{*}(K)$ for all $n \in \mathbb{N}$. Suppose in addition that the generalized commutant $\left\{L_{k}, L_{k}{ }^{*}, k \in \mathbb{N}\right\}^{\prime}$ is reduced to $\mathbb{C} \mathbf{1}$.

If there exists a faithful stationary probability on $\mathbf{S p}(K)$ for the reduced semigroup, then the QMS is ergodic and $K$ induces decoherence of $\mathcal{T}$.

Proof. By Theorem $6, p(K)$ defines a faithful normal stationary state for the semigroup $\mathcal{T}$ so that, from one hand, $K$ induces decoherence of $\mathcal{T}$ and in addition, the results on ergodicity of [15] can be applied. Since $\mathfrak{F}(\mathcal{T}) \subseteq$ $\mathfrak{N}(\mathcal{T}) \subseteq\left\{L_{k}, L_{k}{ }^{*}, k \in \mathbb{N}\right\}^{\prime}$ and the latter is trivial, one obtains $\mathfrak{F}(\mathcal{T})=\mathfrak{N}(\mathcal{T})=\mathbb{C} 1$, so that $\mathcal{T}$ is ergodic after Theorem II.1 in [15].

\subsection{Examples}

We come back to our well-known examples.

\subsubsection{The harmonic oscillator}

Clearly the hypothesis of the last corollary apply here. The algebra generated by $a, a^{\dagger}$ and $\mathbf{1}$ is topologically irreducible, that means that the commutant is $\mathbb{C} 1$. The birth and death semigroup has a faithful invariant probability measure $p$ since $\lambda_{n}<\mu_{n}$, for all $n$. So that $H$ induces decoherence of the semigroup $\mathcal{T}$.

\subsubsection{The quantum exclusion process}

Here, the elements of the orthonormal basis are denoted $|\eta\rangle$ according to the notations introduced in the second quantization procedure. We summarize below the application of the previous corollaries to this model. Consider first a density matrix which is of the form $p(H)$, that is:

$$
\rho=\sum_{\eta} p(\eta)|\eta\rangle\langle\eta|
$$


where $\eta \mapsto p(\eta)$ is a summable function with $\sum_{\eta} p(\eta)=1$.

Proposition 5. Let assume that

$$
\pi(i) \gamma_{i, j}=\pi(j) \gamma_{j, i} \quad(i, j \in \mathbb{N})
$$

where $(\pi(i))_{i \in \mathbb{N}}$ is any sequence of positive numbers. Then a normal state $\omega$ with density matrix $\rho$ given by (36) is stationary if

$$
p(\eta)=\prod_{i \in \mathbb{N}} \alpha_{i}(\eta(i)),
$$

for all $\eta \in \mathbf{S}$, where $\alpha_{i}:\{0,1\} \rightarrow[0,1]$ is, for each $i \in \mathbb{N}$, a probability measure given by

$$
\alpha_{i}(x)=\frac{(\pi(i))^{x}}{1+\pi(i)} \quad(i \in \mathbb{N}, x \in\{0,1\}) .
$$

Moreover, $H$ induces decoherence of the semigroup $\mathcal{T}$.

Proof. After proving the classical reduction of the semigroup, the expression of the stationary probability $p(\eta)$ is indeed a straightforward consequence of Theorem 2.1 in Chapter VIII of [23], however, we provide here a direct proof for the sake of completeness. We first notice that given the probabilities $\alpha_{i}$ on $\{0,1\}$, by Kolmogorov's Theorem there is a unique probability measure $\mathbf{P}_{\alpha}$ on the set of configurations $\mathbf{S}$ with marginals

$$
\mathbf{P}_{\alpha}(\{\eta \in \mathbf{S}: \eta(i)=1 \text {, for all } i \in I ; \eta(j)=0 \text {, for all } j \in J\})=\prod_{i \in I} \alpha_{i}(1) \prod_{j \in J} \alpha_{j}(0) .
$$

And $p(\eta)=\mathbf{P}_{\alpha}(\{\eta\})$.

Let be given $\rho$ by (36). Then $\mathcal{L}_{*}(\rho)=0$ if and only if $\operatorname{tr}\left(\mathcal{L}_{*}(\rho)|\eta\rangle\langle\eta|\right)=0$ for all $\eta \in \mathbf{S}$.

Notice that $\zeta-1_{j}+1_{i}=\eta$ if and only if $\zeta=\eta-1_{i}+1_{j}$, for $\eta, \zeta \in \mathbf{S}, i, j \in \mathbb{N}$. Thus, if we write

$$
\rho=\sum_{\zeta} p(\zeta)|\zeta\rangle\langle\zeta|
$$

the previous theorem yields

$$
\mathcal{L}_{*}(\rho)=\sum_{\zeta} \sum_{i, j: i \stackrel{\zeta}{\rightarrow} j} \gamma_{i, j} p(\zeta)\left(\left|\zeta-1_{i}+1_{j}\right\rangle\left\langle\zeta-1_{i}+1_{j}|-| \zeta\right\rangle\langle\zeta|\right) .
$$

Now, $\operatorname{tr}\left(\left|\zeta-1_{i}+1_{j}\right\rangle\left\langle\zeta-1_{i}+1_{j}|| \eta\right\rangle\langle\eta|\right)=1$ if and only if $\zeta=\eta-1_{j}+1_{i}$. Thus, $\operatorname{tr}\left(\mathcal{L}_{*}(\rho)|\eta\rangle\langle\eta|\right)=0$ if and only if

$$
\sum_{i, j}(1-\eta(i)) \eta(j)\left(\gamma_{i, j} p\left(\eta-1_{j}+1_{i}\right)-\gamma_{j, i} p(\eta)\right)=0 .
$$

Notice that $\alpha_{i}(0) p\left(\eta+1_{i}\right)=p(\eta) \alpha_{i}(1)$ and $\alpha_{j}(1) p\left(\eta-1_{j}\right)=p(\eta) \alpha_{j}(0)$. These equations yields

$$
\gamma_{i, j} p\left(\eta-1_{j}+1_{i}\right)-\gamma_{j, i} p(\eta)=\left(\gamma_{i, j} \frac{\pi(i)}{\pi(j)}-\gamma_{j, i}\right) p(\eta)
$$

and the last term between brackets is zero, by the hypothesis (37). The last expression implies that $L p(\eta)=0$. Moreover the above computations yield

$$
\mathcal{L}_{*}(|\eta\rangle\langle\eta|)=\sum_{i, j} c_{i, j}(\eta)\left(\left|\eta-1_{i}+1_{j}\right\rangle\left\langle\eta-1_{i}+1_{j}|-| \eta\right\rangle\langle\eta|\right),
$$

which commutes with $H$, so that Theorem 6 applies and the proof is complete. 
The above result can be rephrased in a slightly different framework to recover a unique faithful stationary state in the Gibbs form. We add a cemetery to the classical Markov chain by completing $\mathbb{N}$ with a point $\infty \notin \mathbb{N}$. We assume that $\gamma_{i, \infty}, \gamma_{\infty, j}>0$ but $\gamma_{\infty, \infty}=0$. On the other hand, we put $L_{i, \infty}=\sqrt{\gamma_{i, \infty}} b_{i}, L_{\infty, j}=\sqrt{\gamma_{\infty}, j} b_{j}^{\dagger}$, for all $i, j \in \mathbb{N}$, and $L_{\infty, \infty}=0$. Configurations are now defined on $\overline{\mathbb{N}}=\mathbb{N} \cup\{\infty\}$. Finally define $E_{\infty}=\mu>0$, which we call the chemical potential. The generator $\mathcal{L}(\cdot)$ is naturally extended taking the sum in (30) running over all indexes $(i, j) \in \overline{\mathbb{N}} \times \overline{\mathbb{N}}$. Moreover, this time the set $\left\{L_{i, j}, L_{i, j}^{*} ; i, j \in \overline{\mathbb{N}}\right\}$ includes all the operators $b^{\dagger}(k), b(k)$ generating the CAR algebra, so that its generalized commutant algebra is trivial. Thus, in this case we have $\mathfrak{F}(\mathcal{T})=\mathfrak{N}(\mathcal{T})=\mathbb{C} 1$ and the semigroup is ergodic as soon as we provide a faithful stationary state.

In the following we assume $\beta>0$, and remind that $H$ is supposed to be bounded from below. A straightforward computation yields

$$
\operatorname{tr}\left(\mathrm{e}^{-\beta(H-\mu N)}\right)=\prod_{i \in \mathbb{N}}\left(1+\mathrm{e}^{-\beta\left(E_{i}-\mu\right)}\right)<\infty .
$$

Corollary 5. Assume that the Hamiltonian $H$ given by (18) is bounded from below. Moreover, suppose that (37) is satisfied with

$$
\pi(i)=\mathrm{e}^{-\beta\left(E_{i}-\mu\right)} \quad(i \in \overline{\mathbb{N}}),
$$

where $\beta>0$. Denote

$$
Z(\beta, \mu)=\operatorname{tr}\left(\mathrm{e}^{-\beta(H-\mu N)}\right) .
$$

Then

$$
\rho=\frac{1}{Z(\beta, \mu)} \mathrm{e}^{-\beta(H-\mu N)},
$$

is a faithful stationary state of the quantum Markov semigroup associated to $H$ and the operators $L_{\ell}$ considered in the previous results. The semigroup is ergodic and $H$ induces decoherence of $\mathcal{T}$.

Remark 10. As a final remark, it is worth noticing that if a quantum Markov semigroup is transient, there is no stationary state. As a result, decoherence as defined here cannot take place. As an example of a transient QMS, the reader is referred to [18] where it is showed that the semigroup of a quantum Brownian motion for $d \geqslant 2$ is transient.

\section{Remerciements}

Le moment est venu de remercier. De remercier tout d'abord Paul-André Meyer pour son enseignement, pour ses oeuvres, pour ses questions fondamentales débordant le cadre mathématique, pour la vie qu'il a crée en fin, tout simplement, pour avoir existé.

Le colloque en mémoire de Paul André, réunissant des probabilistes de plusieurs générations, a été l'occasion de retrouvailles émouvantes. Nous avons tous partagé l'émotion du souvenir et l'intérêt des recherches du présent. Cela a été rendu possible grâce à l'excellent travail du comité organisateur du colloque à qui j'addresse ici ma gratitude. Tout particulièrement, je remercie Michel Emery et Jacques Franchi pour leur chalereux accueil qui fait honneur à la traditionnelle hospitalité de l'Ecole de Probabilités de Strasbourg.

\section{References}

[1] L. Accardi, Y.G. Lu, I. Volovich, Quantum Theory and its Stochastic Limit, Springer-Verlag, 2002.

[2] R. Alicki, K. Lendi, Quantum Dynamical Semigroups and Applications, Lecture Notes in Phys., vol. 286, Springer-Verlag, 1987. 
[3] Ph. Biane, Quelques propriétés du mouvement Brownien non-commutatif. Hommage à P.A. Meyer et J. Neveu, Astérisque 236 (1996) 73-102.

[4] Ph. Blanchard, R. Olkiewicz, Decoherence induced transition from quantum to classical dynamics, Rev. Math. Phys. 15 (2003) $217-243$.

[5] O. Bratteli, D.W. Robinson, Operator Algebras and Quantum Statistical Mechanics, vol. 1, second ed., Springer-Verlag, 1987.

[6] O. Bratteli, D.W. Robinson, Operator Algebras and Quantum Statistical Mechanics, vol. 2, second ed., Springer-Verlag, 1996.

[7] M. Brune, et al., Phys. Rev. Lett. 77 (1996) 4887.

[8] A.M. Chebotarev, F. Fagnola, Sufficient conditions for conservativity of minimal quantum dynamical semigroups, J. Funct. Anal. 153 (1998) 382-404.

[9] A.M. Chebotarev, Lectures on Quantum Probability, Aportaciones Matemáticas, Ser. Textos, vol. 14, Soc. Mat. Mexicana, México, 2000.

[10] E. Christensen, D.E. Evans, Cohomology of operator algebras and quantum dynamical semigroups, J. London Math. Soc. 20 (1979) 358-368.

[11] E.B. Davies, Quantum dynamical semigroups and the neutron diffusion equation, Rep. Math. Phys. 11 (1977) 169-188.

[12] C. Dellacherie, P.-A. Meyer, Probabilités et potentiel. Chapitres XII-XVI, second ed., Hermann, Paris, 1987.

[13] G. Dell Antonio, On Decoherence, J. Math. Phys. 44 (2003) 4939-4956.

[14] F. Fagnola, Quantum Markov semigroups and quantum flows, Proyecciones J. Math. 18 (3) (1999) 1-144.

[15] F. Fagnola, R. Rebolledo, The approach to equilibrium of a class of quantum dynamical semigroups, Infinite Dimensional Anal. Quantum Probab. Related Topics 1 (4) (1998) 1-12.

[16] F. Fagnola, R. Rebolledo, On the existence of invariant states for quantum dynamical semigroups, J. Math. Phys. 42 (2001) $1296-1308$.

[17] F. Fagnola, R. Rebolledo, Subharmonic projections for a quantum Markov semigroup, J. Math. Phys. 43 (2002) $1074-1082$.

[18] F. Fagnola, R. Rebolledo, Transience and recurrence of quantum Markov semigroups, Probab. Theory Related Fields 126 (2003) $289-306$.

[19] A. Frigerio, M. Verri, Long-time asymptotic properties of dynamical semigroups on $w^{*}$-algebras, Math. Z. (1982).

[20] D. Giulini, et al., Decoherence and the Appearance of a Classical world in Quantum Theory, Springer, Heidelberg, 1996.

[21] V. Gorini, A. Kossakowski, E.C.G. Sudarshan, Completely positive dynamical semigroups of n-level systems, J. Math. Phys. 17 (1976) 821-825.

[22] A. Holevo, Statistical Structure of Quantum Theory, Lecture Notes in Phys., vol. 67, Springer-Verlag, 2001.

[23] T.M. Liggett, Interacting Particle Systems, Springer-Verlag, New York, 1985.

[24] T.M. Liggett, Stochastic Interacting Systems: Contact, Voter and Exclusion Processes, Springer-Verlag, Berlin, 1999.

[25] G. Lindblad, On the generators of quantum dynamical semigroups, Commun. Math. Phys. 48 (1976) 119-130.

[26] A. Majewski, R.F. Streater, Detailed balance and quantum dynamical maps, J. Phys. A 31 (1998) 7981-7995.

[27] P.-A. Meyer, Quantum Probability for Probabilists, Lecture Notes in Math., vol. 1538, Springer-Verlag, Berlin, 1993.

[28] C.J. Myatt, et al., Nature 403 (2000) 269.

[29] A. Pazy, Semigroups of Linear Operators and Applications to Partial Differential Equations, Springer-Verlag, 1983.

[30] K.R. Parthasarathy, An Introduction to Quantum Stochastic Calculus, Monographs in Math., vol. 85, Birkhaüser, Basel, 1992.

[31] W.F. Stinespring, Positive functions on $C^{*}$-algebras, Proc. Amer. Math. Soc. 6 (1955) 211-216.

[32] G.K. Pedersen, Analysis Now, Graduate Texts in Math., vol. 118, Springer-Verlag, 1989.

[33] W.T. Strunz, Decoherence in quantum physics, in: A. Buchleitner, K. Hornberger (Eds.), Coherent Evolution in Noisy Environments, in: Lecture Notes in Phys., vol. 611, Springer-Verlag, 2002, pp. 199-233. 